AperTO - Archivio Istituzionale Open Access dell'Università di Torino

\title{
Nested Sequent Calculi for Normal Conditional Logics
}

\section{This is the author's manuscript}

Original Citation:

Availability:

This version is available http://hdl.handle.net/2318/134107

since 2017-05-28T18:43:28Z

Published version:

DOI:10.1093/logcom/ext034

Terms of use:

Open Access

Anyone can freely access the full text of works made available as "Open Access". Works made available under a Creative Commons license can be used according to the terms and conditions of said license. Use of all other works requires consent of the right holder (author or publisher) if not exempted from copyright protection by the applicable law. 


\title{
Nested Sequent Calculi for Normal Conditional Logics
}

\author{
Régis Alenda $^{1} \quad$ Nicola Olivetti $^{2} \quad$ Gian Luca Pozzato ${ }^{3}$ \\ ${ }^{1}$ Aix-Marseille Université, CNRS, LSIS UMR 7296, 13397, Marseille, France. \\ regis.alenda@univ-amu.fr \\ ${ }^{2}$ Aix-Marseille Université, CNRS, LSIS UMR 7296, 13397, Marseille, France. \\ nicola.olivetti@univ-amu.fr \\ ${ }^{3}$ Dipartimento di Informatica - Universitá di Torino, Italy. \\ gianluca.pozzato@unito.it
}

\begin{abstract}
Nested sequent calculi are a useful generalization of ordinary sequent calculi, where sequents are allowed to occur within sequents. Nested sequent calculi have been profitably employed in the area of (multi)-modal logic to obtain analytic and modular proof systems for these logics. In this work, we extend the realm of nested sequents by providing nested sequent calculi for the basic conditional logic $\mathrm{CK}$ and some of its significant extensions. We provide also a calculus for Kraus Lehman Magidor cumulative logic C. The calculi are internal (a sequent can be directly translated into a formula), cut-free and analytic. Moreover, they can be used to design (sometimes optimal) decision procedures for the respective logics, and to obtain complexity upper bounds. Our calculi are an argument in favour of nested sequent calculi for modal logics and alike, showing their versatility and power.
\end{abstract}

\section{Introduction}

The recent history of conditional logics starts with the work by Lewis [25, 26], who proposed them in order to formalize a kind of hypothetical reasoning (if $A$ were the case then $B$ ), that cannot be captured by classical logic with material implication. One original motivation was to formalize counterfactual sentences, i.e. conditionals of the form "if $A$ were the case then $B$ would be the case", where $A$ is false. Conditional logics have found an interest in several fields of artificial intelligence and knowledge representation. They have been used to reason about prototypical properties [16] and to model belief change $[21,18]$. Moreover, conditional logics can provide an axiomatic foundation of nonmonotonic reasoning [5, 22], here a conditional $A \Rightarrow B$ is read as "in normal circumstances if $A$ then $B$ ". Recently, constructive conditional logics have been applied to reason about access control policies [14, 13]: the statement $A$ says $B$, intuitively meaning that a user/a program $A$ asserts $B$ to hold in the system, can be naturally expressed by a conditional $A \Rightarrow B$. Finally, a kind of (multi)-conditional logics $[3,4]$ have been used to formalize epistemic change in a multi-agent setting and 
in some kind of epistemic "games", each conditional operator expresses the "conditional beliefs" of an agent.

Semantically, all conditional logics enjoy a possible world semantics, with the intuition that a conditional $A \Rightarrow B$ is true in a world $x$ if $B$ is true in the set of worlds where $A$ is true and that are most similar to/closest to/"as normal as" $x$. Since there are different ways of formalizing "the set of worlds similar/closest/..." to a given world, there are expectedly rather different semantics for conditional logics, from the most general selection function semantics to the stronger sphere semantics.

The selection function semantics $[31,26]$ is based on possible world structures equipped by a selection function $f$. Truth values are assigned to formulas depending on a world; intuitively, the selection function $f$ selects, for a world $w$ and a formula $A$, the set of worlds $f(w, A)$ which are "most-similar to $w$ " or "closer to $w$ " given the information $A$. Considering this semantics, $\mathrm{CK}$ is the fundamental system; it has the same role as the system $\mathrm{K}$ (from which it derives its name) in modal logic: CK-valid formulas are exactly those ones that are valid in every selection function model. Exactly as in modal logics, extensions of the basic system CK are obtained by restricting the attention to models whose selection function satisfies specific properties. Just to mention a few, the following is a non exhaustive list of extensions of CK known in the literature [26]:

\begin{tabular}{lll} 
Axiom name & Abbreviation & Axiom \\
\hline & & \\
Conditional Excluded Middle & CEM & $(A \Rightarrow B) \vee(A \Rightarrow \neg B)$ \\
Identity & ID & $A \Rightarrow A$ \\
Conditional Modus Ponens & MP & $(A \Rightarrow B) \rightarrow(A \rightarrow B)$ \\
Conditional Strong centering & CS & $(A \wedge B) \rightarrow(A \Rightarrow B)$ \\
Conditional Substitution & CSO & $(A \Rightarrow B) \wedge(B \Rightarrow A) \rightarrow((A \Rightarrow C) \rightarrow(B \Rightarrow C))$ \\
Conditional Additivity & CA & $(A \Rightarrow B) \wedge(C \Rightarrow B) \rightarrow(A \vee C \Rightarrow B)$ \\
Cumulative Monotony & CM & $(A \Rightarrow B) \wedge(A \Rightarrow C) \rightarrow(A \wedge C \Rightarrow B)$ \\
Restricted Transitivity & RT & $(A \wedge B \Rightarrow C) \rightarrow((A \Rightarrow B) \rightarrow(A \Rightarrow C))$ \\
Modal & MOD & $(A \Rightarrow \perp) \rightarrow(B \Rightarrow \neg A)$ \\
Conditional variability & CV & $(A \Rightarrow C) \wedge \neg(A \Rightarrow \neg B) \rightarrow(A \wedge B \Rightarrow C)$
\end{tabular}

From the point of view of proof-theory and automated deduction, conditional logics do not have however a state of the art comparable with, say, the one of modal logics, where there are well-established alternative calculi, whose proof-theoretical and computational properties are well-understood. This is partially due to the mentioned lack of a unifying semantics; as a matter of fact the most general semantics, the selection function one, is of little help for proof-theory, and the preferential/sphere semantics only captures a subset of (actually rather strong) systems. Similarly to modal logics and other extensions/alternative to classical logics two types of calculi have been studied: external calculi which make use of labels and relations on them to import the semantics into the syntax, and internal calculi which stay within the language, so that a "configuration" (sequent, tableaux node...) can be directly interpreted as a formula of the language. Just to mention some work, to the first stream belongs [2] proposing a calculus for (unnested) cumulative logic $\mathbf{C}$ (see below). More recently, [28] presents modular labeled calculi (of optimal complexity) for $\mathrm{CK}$ and some of its extensions, basing on the selection function semantics, and [19] presents modular labeled calculi for preferential logic PCL and its extensions. The latter calculi take advantage of a sort of hybrid modal translation. To the second stream belong the calculi by Gent [15] and 
by de Swart [32] for Lewis' logic $\mathbb{V} \mathbb{C}$ and neighbours ${ }^{1}$. These calculi manipulate sets of formulas and provide a decision procedure, although they comprise an infinite set of rules. Very recently, some internal calculi for CK and some extensions (with any combination of MP, ID, CEM) have been proposed by Pattinson and Schröder [29] The calculi are obtained by a general method for closing a set of rules (corresponding to Hilbert axioms) with respect to the cut rule. These calculi have optimal complexity; notice that some of the rules do not have a fixed number of premises. These calculi have been extended to preferential conditional logics [30], i.e. including cumulativity (CM) and the or-axiom (CA), although the resulting systems are fairly complicated. Finally in [24] the authors provide internal calculi for Lewis' conditional logic $\mathbb{V}$ and some extensions. Their calculi are formulated for a language comprising the entrenchment connective, the strong and the weak conditional operator, both conditional operators can be defined in terms of the entrenchment connective. These calculi are actually an extension of Gent's and de Swart's ones. Basing on these calculi, the authors obtain a PSPACE decision procedure for the considered logics.

In this paper we begin to investigate nested sequents calculi for conditional logics. Nested sequents calculi are a natural generalization of ordinary sequent calculi where sequents are allowed to occur within sequents. However a nested sequent always corresponds to a formula of the language, so that we can think of the rules as operating "inside a formula", combining subformulas rather than just combining outer occurrences of formulas as in ordinary sequent calculi. In this sense, they are a special kind of "deep inference" calculi as proposed by Guglielmi and colleagues [10,9]. Nested calculi have been provided for modal logics by Brünnler $[7,6,8]$ and extended further by Fitting [12] who has also clarified their relations with prefixed tableaux. In [20] nested sequent calculi have been provided for Bi-Intuitionistic Logic.

In this paper we treat the basic normal conditional logic $\mathrm{CK}$ and its extensions with ID, MP and CEM. We also consider cumulative logic $\mathbf{C}$ introduced in [22] which corresponds to the flat fragment (i.e., without nested conditionals) of $\mathrm{CK}+\mathrm{CSO}+\mathrm{ID}$. The calculi are rather natural, all rules have a fixed number of premises. The completeness is established by cut-elimination, whose peculiarity is that it must take into account the substitution of equivalent antecedents of conditionals (a condition corresponding to normality). The calculi can be used to obtain a decision procedure for the respective logics by imposing some restrictions preventing redundant applications of rules. In all cases, we get a PSPACE upper bound, a bound that for CK and its extensions with ID and MP is optimal (but not for CK+CEM that is known to be CONP). For flat $\mathrm{CK}+\mathrm{CSO}+\mathrm{ID}=$ cumulative logic $\mathbf{C}$ we also get a PSPACE bound, we are not aware of a better upper bound for this logic (although we may suspect that it is not optimal). We can see the present work as a further argument in favor of nested sequents as a useful tool to provide natural, yet computationally adequate, calculi for modal extensions of classical logic.

This paper extends and revises preliminary results proposed in [1].

${ }^{1}$ An axiomatization of Lewis' logic $\mathbb{V} \mathbb{C}$ is provided in [27] as a combination of axioms ID, MOD, CSO, $\mathrm{CV}$, MP, and CS. 


\section{Conditional Logics}

A propositional conditional language $\mathcal{L}$ contains:

- a set of propositional variables $A T M$;

- the symbols of false $\perp$ and true $\top$;

- a set of connectives $\wedge, \vee, \neg, \rightarrow, \Rightarrow$.

We define formulas of $\mathcal{L}$ as follows:

- $\perp, \top$ and the propositional variables of $A T M$ are atomic formulas;

- if $A$ and $B$ are formulas, then $\neg A$ and $A \otimes B$ are complex formulas, where $\otimes \in\{\wedge, \vee, \rightarrow, \Rightarrow\}$.

We adopt the selection function semantics. We consider a non-empty set of possible worlds $\mathcal{W}$. Intuitively, the selection function $f$ selects, for a world $w$ and a formula $A$, the set of worlds of $\mathcal{W}$ which are closest to $w$ given the information $A$. A conditional formula $A \Rightarrow B$ holds in a world $w$ if the formula $B$ holds in all the worlds selected by $f$ for $w$ and $A$.

Definition 1 (Selection function semantics) A model is a triple

$$
\mathcal{M}=\langle\mathcal{W}, f,[]\rangle
$$

where:

- $\mathcal{W}$ is a non empty set of objects called worlds;

- $f$ is the selection function $f: \mathcal{W} \times 2^{\mathcal{W}} \longmapsto 2^{\mathcal{W}}$;

- [ ] is the evaluation function, which assigns to an atom $P \in A T M$ the set of worlds where $P$ is true, and is extended to boolean formulas as follows:

$$
\begin{aligned}
& {[\top]=\mathcal{W}} \\
& {[\perp]=\emptyset} \\
& {[\neg A]=\mathcal{W}-[A]} \\
& {[A \wedge B]=[A] \cap[B]} \\
& {[A \vee B]=[A] \cup[B]} \\
& {[A \rightarrow B]=[B] \cup(\mathcal{W}-[A])} \\
& {[A \Rightarrow B]=\{w \in \mathcal{W} \mid f(w,[A]) \subseteq[B]\}}
\end{aligned}
$$

A formula $F \in \mathcal{L}$ is valid in a model $\mathcal{M}=\langle\mathcal{W}, f,[]\rangle$, and we write $\mathcal{M} \models F$, if $[F]=\mathcal{W}$. A formula $F \in \mathcal{L}$ is valid, and we write $\models F$, if it is valid in every model, that is to say $\mathcal{M} \models F$ for every $\mathcal{M}$.

We have defined $f$ taking $[A]$ rather than $A$ (i.e. $f(w,[A])$ rather than $f(w, A)$ ) as an argument; this is equivalent to define $f$ on formulas, i.e. $f(w, A)$ but imposing that if $[A]=\left[A^{\prime}\right]$ in the model, then $f(w, A)=f\left(w, A^{\prime}\right)$. This condition is called normality.

The semantics above characterizes the basic conditional system, called CK [26]. An axiomatization of $\mathrm{CK}$ is given by ( $\vdash$ denotes provability in the axiom system): 
- any axiomatization of the classical propositional calculus

(prop)

- If $\vdash A$ and $\vdash A \rightarrow B$, then $\vdash B$

(Modus Ponens)

- If $\vdash A \leftrightarrow B$ then $\vdash(A \Rightarrow C) \leftrightarrow(B \Rightarrow C)$

(RCEA)

- If $\vdash\left(A_{1} \wedge \cdots \wedge A_{n}\right) \rightarrow B$ then $\vdash\left(C \Rightarrow A_{1} \wedge \cdots \wedge C \Rightarrow A_{n}\right) \rightarrow(C \Rightarrow B)$ (RCK)

Other conditional systems are obtained by assuming further properties on the selection function. In this work, we consider the following standard extensions of the basic system CK:

\begin{tabular}{|l|l|l|}
\hline System & Axiom & Model condition \\
\hline ID & $A \Rightarrow A$ & $f(w,[A]) \subseteq[A]$ \\
CEM & $(A \Rightarrow B) \vee(A \Rightarrow \neg B)$ & $|f(w,[A])| \leq 1$ \\
MP & $(A \Rightarrow B) \rightarrow(A \rightarrow B)$ & $w \in[A]$ implies $w \in f(w,[A])$ \\
CSO & $(A \Rightarrow B) \wedge(B \Rightarrow A) \rightarrow$ & $f(w,[A]) \subseteq[B]$ and $f(w,[B]) \subseteq[A]$ \\
& $((A \Rightarrow C) \rightarrow(B \Rightarrow C))$ & implies $f(w,[A])=f(w,[B])$ \\
\hline
\end{tabular}

The above axiomatization is sound and complete with respect to the semantics [26]:

Theorem 2 (Soundness and completeness of axiomatization w.r.t. the semantics of Definition 1 [26]) Given a formula $F \in \mathcal{L}, F$ is valid in a conditional logic if and only if it is provable in the respective axiomatization, i.e. $\models F$ if and only if $\vdash F$.

\section{Nested Sequent Calculi $\mathcal{N} S$ for Conditional Logics}

In this section we present nested sequent calculi $\mathcal{N} S$, where $S$ is an abbreviation for $\mathrm{CK}\{+\mathrm{X}\}$, and $\mathrm{X}=\{\mathrm{CEM}, \mathrm{ID}, \mathrm{MP}, \mathrm{ID}+\mathrm{MP}, \mathrm{CEM}+\mathrm{ID}\}$. We are able to deal with the basic normal conditional logic CK and its extensions with axioms ID, MP and CEM. We are also able to deal with some combinations of them, namely the systems allowing ID with either MP or CEM. The problem of extending $\mathcal{N} S$ to the conditional logics allowing both MP and CEM is open at present. As usual, the completeness of the calculi is an easy consequence of the admissibility of cut. We are also able to turn $\mathcal{N} S$ into a terminating calculus, which gives us a decision procedure for the respective conditional logics.

Definition 3 A nested sequent $\Gamma$ is defined inductively as follows:

- a formula of $\mathcal{L}$ is a nested sequent;

- if $A$ is a formula and $\Gamma$ is a nested sequent, then $[A: \Gamma]$ is a nested sequent;

- a finite multiset of nested sequents is a nested sequent.

A nested sequent can be displayed as

$$
A_{1}, \ldots, A_{m},\left[B_{1}: \Gamma_{1}\right], \ldots,\left[B_{n}: \Gamma_{n}\right],
$$

where $n, m \geq 0, A_{1}, \ldots, A_{m}, B_{1}, \ldots, B_{n}$ are formulas and $\Gamma_{1}, \ldots, \Gamma_{n}$ are nested sequents.

First of all, let us define the depth of a nested sequent: 
Definition 4 (Depth of a nested sequent) The depth $d(\Gamma)$ of a nested sequent $\Gamma$ is defined as follows:

- if $\Gamma=A$, then $d(\Gamma)=0$;

- if $\Gamma=[A: \Delta]$, then $d(\Gamma)=1+d(\Delta)$

- if $\Gamma=\Gamma_{1}, \Gamma_{2}, \ldots, \Gamma_{n}$, then $d(\Gamma)=\max \left(d\left(\Gamma_{1}\right), d\left(\Gamma_{2}\right), \ldots, d\left(\Gamma_{n}\right)\right)$.

where $A, A_{1}, A_{2}, \ldots, A_{m}$ are formulas of $\mathcal{L}$ and $\Gamma, \Gamma_{1}, \Gamma_{2}, \ldots, \Gamma_{n}$ are nested sequents.

A nested sequent can be directly interpreted as a formula, just replace "," by $\vee$ and “:” by $\Rightarrow$. More explicitly, the interpretation of a nested sequent

$$
A_{1}, \ldots, A_{m},\left[B_{1}: \Gamma_{1}\right], \ldots,\left[B_{n}: \Gamma_{n}\right]
$$

is inductively defined by the formula

$$
\mathcal{F}(\Gamma)=A_{1} \vee \ldots \vee A_{m} \vee\left(B_{1} \Rightarrow \mathcal{F}\left(\Gamma_{1}\right)\right) \vee \ldots \vee\left(B_{n} \Rightarrow \mathcal{F}\left(\Gamma_{n}\right)\right)
$$

For example, the nested sequent

$$
A, B,[A: C,[B: E, F]],[A: D]
$$

denotes the formula

$$
A \vee B \vee(A \Rightarrow C \vee(B \Rightarrow(E \vee F))) \vee(A \Rightarrow D)
$$

The specificity of nested sequent calculi is to allow inferences that apply within formulas. In order to introduce the rules of the calculus, we need the notion of context. Intuitively a context denotes a "hole", a unique empty position, within a sequent that can be filled by a sequent. We use the symbol ( ) to denote the empty context. A context is defined inductively as follows:

Definition 5 We define a context:

- $\Gamma()=\Delta,()$ is a context with depth $d(\Gamma())=0$;

- if $\Sigma()$ is a context, $\Gamma()=\Delta,[A: \Sigma()]$ is a context with depth $d(\Gamma())=$ $1+d(\Sigma())$.

Finally we define the result of filling "the hole" of a context by a sequent:

Definition 6 Let $\Gamma($ ) be a context and $\Delta$ be a sequent, then the sequent obtained by filling the context by $\Delta$, denoted by $\Gamma(\Delta)$ is defined as follows:

- if $\Gamma()=\Lambda,($ ), then $\Gamma(\Delta)=\Lambda, \Delta$;

- if $\Gamma()=\Lambda,[A: \Sigma()]$, then $\Gamma(\Delta)=\Lambda,[A: \Sigma(\Delta)]$. 
The calculi $\mathcal{N} S$ are shown in Figure 1. As usual, we say that a nested sequent $\Gamma$ is derivable in $\mathcal{N} S$ if it admits a derivation. A derivation is a tree whose nodes are nested sequents. A branch is a sequence of nodes $\Gamma_{1}, \Gamma_{2}, \ldots, \Gamma_{n}, \ldots$ Each node $\Gamma_{i}$ is obtained from its immediate successor $\Gamma_{i-1}$ by applying backward a rule of $\mathcal{N} S$, having $\Gamma_{i-1}$ as the conclusion and $\Gamma_{i}$ as one of its premises. A branch is closed if one of its nodes is an instance of axioms $(A X),\left(A X_{\top}\right),\left(A X_{\perp}\right)$, otherwise it is open. We say that a tree is closed if all its branches are closed. A nested sequent $\Gamma$ has a derivation in $\mathcal{N} S$ if there is a closed tree having $\Gamma$ as a root. As an example, Figure 2 shows a derivation of (an instance of) the axiom ID.

Before analyzing in detail the calculi $\mathcal{N} S$, let us define the complexity of a formula $F$ of $\mathcal{L}$, that will be used in the proof of several properties of the calculi themselves.

Definition 7 (Complexity of a formula) Given a formula $F \in \mathcal{L}$, we define the complexity $c p(F)$ as follows:

- $c p(P)=1$, if $P \in A T M$

- $c p(\top)=1$

- $c p(\perp)=1$

- $c p(\neg A)=1+c p(A)$

- $c p(A \otimes B)=1+c p(A)+c p(B)$, where $\otimes \in\{\wedge, \vee, \rightarrow, \Rightarrow\}$.

$$
\begin{aligned}
& \begin{array}{lrr}
\Gamma(P, \neg P) \underset{P \in A T M}{(A X)} & \Gamma(\top)\left(A X_{\top}\right) & \Gamma(\neg \perp) \quad\left(A X_{\perp}\right) \\
\frac{\Gamma(A)}{\Gamma(\neg \neg A)}(\neg) & \frac{\Gamma(A) \quad \Gamma(B)}{\Gamma(A \wedge B)}\left(\wedge^{+}\right) & \frac{\Gamma(\neg A, \neg B)}{\Gamma(\neg(A \wedge B))}\left(\wedge^{-}\right)
\end{array} \\
& \frac{\Gamma(A, B)}{\Gamma(A \vee B)}\left(\vee^{+}\right) \quad \frac{\Gamma(\neg A) \quad \Gamma(\neg B)}{\Gamma(\neg(A \vee B))}\left(\vee^{-}\right) \quad \frac{\Gamma(\neg A, B)}{\Gamma(A \rightarrow B)}\left(\rightarrow^{+}\right) \quad \frac{\Gamma(A) \quad \Gamma(\neg B)}{\Gamma(\neg(A \rightarrow B))}\left(\rightarrow^{-}\right) \\
& \frac{\Gamma([A: B])}{\Gamma(A \Rightarrow B)}\left(\Rightarrow^{+}\right) \quad \frac{\Gamma\left(\neg(A \Rightarrow B),\left[A^{\prime}: \Delta, \neg B\right]\right) \quad A, \neg A^{\prime} \quad A^{\prime}, \neg A}{\Gamma\left(\neg(A \Rightarrow B),\left[A^{\prime}: \Delta\right]\right)}\left(\Rightarrow^{-}\right) \quad \frac{\Gamma([A: \Delta, \neg A])}{\Gamma([A: \Delta])}(I D) \\
& \frac{\Gamma(\neg(A \Rightarrow B), A) \quad \Gamma(\neg(A \Rightarrow B), \neg B)}{\Gamma(\neg(A \Rightarrow B))}(M P) \quad \frac{\Gamma([A: \Delta, \Sigma],[B: \Sigma]) \quad A, \neg B \quad B, \neg A}{\Gamma([A: \Delta],[B: \Sigma])}(C E M)
\end{aligned}
$$

Figure 1: The nested sequent calculi $\mathcal{N} S$.

The following lemma shows that axioms can be generalized to any formula $F$ :

Lemma 8 Given any formula $F$ and any context $\Gamma()$, the sequent $\Gamma(F, \neg F)$ is derivable in $\mathcal{N} S$. 


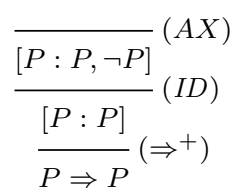

Figure 2: A derivation of the axiom ID.

Proof. By induction on the complexity of $F$. For the base case, we distinguish three subcases: $F$ is either $(i) P \in A T M$ or $(i i) \top$ or $($ iii $) \neg \perp$. Obviously, in all cases $\Gamma(F, \neg F)$ is derivable: in case $(i), \Gamma(F, \neg F)$ is an instance of $(A X)$, in case $(i i)$ it is an instance of $\left(A X_{\top}\right)$, in case $(i i i)$ is an instance of $\left(A X_{\perp}\right)$.

For the inductive step, we apply the inductive hypothesis on the subformula(s) of $F$ and then we conclude by applying the rule(s) manipulating the principal connective in $F$. As an example, consider $F=A \Rightarrow B$. By inductive hypothesis (1) $\Gamma(\neg(A \Rightarrow$ $B),[A: B, \neg B]))$ and (2) $A, \neg A$ are derivable. We conclude as follows:

$$
\frac{(1) \Gamma(\neg(A \Rightarrow B),[A: B, \neg B])) \quad(2) A, \neg A \quad(2) A, \neg A}{\frac{\Gamma([\neg(A \Rightarrow B),[A: B])}{\Gamma(\neg(A \Rightarrow B), A \Rightarrow B)}\left(\Rightarrow^{+}\right)}\left(\Rightarrow^{-}\right)
$$

In [29] the authors propose optimal sequent calculi for CK and its extensions by any combination of ID, MP and CEM. It is not difficult to see that the rules $C K_{g}, C K I D_{g}$, $C K C E M_{g}, C K C E M I D_{g}$ of their calculi are derivable in our calculi.

\subsection{Basic structural properties of $\mathcal{N} S$}

First of all, we show that weakening and contraction are height-preserving admissible in the calculi $\mathcal{N} S$. Furthermore, we show that all the rules of the calculi, with the exceptions of $\left(\Rightarrow^{-}\right)$and $(C E M)$, are height-preserving invertible. However, for $\left(\Rightarrow^{-}\right)$ and $(C E M)$, we can prove a weaker version of invertibility. As usual, we define the height of a derivation as the height of the tree corresponding to the derivation itself.

Lemma 9 (Admissibility of weakening) Weakening is height-preserving admissible in $\mathcal{N} S:$ if $\Gamma(\Delta)$ is derivable in $\mathcal{N} S$ with a derivation of height $h$, then also $\Gamma(\Delta, \Sigma)$ is derivable in $\mathcal{N} S$ with a proof of height $h^{\prime} \leq h$, where $\Delta$ and $\Sigma$ are nested sequents.

Proof. We proceed by induction on the height $h$ of the derivation of $\Gamma(\Delta)$. The base case corresponds to a derivation whose height is 0 , namely $\Gamma(\Delta)$ is either an instance of $(A X)$ or an instance of $\left(A X_{\top}\right)$ or an instance of $\left(A X_{\perp}\right)$, i.e. we are considering a sequent of the form either $\Gamma\left(\Delta^{\prime}, P, \neg P\right)$ or $\Gamma\left(\Delta^{\prime}, \top\right)$ or $\Gamma\left(\Delta^{\prime}, \neg \perp\right)$. We can immediately conclude that also $\Gamma\left(\Delta^{\prime}, P, \neg P, \Sigma\right)$ or $\Gamma\left(\Delta^{\prime}, \top, \Sigma\right)$ or $\Gamma\left(\Delta^{\prime}, \neg \perp, \Sigma\right)$ are instances of axioms, and we are done.

For the inductive step, we have to consider the last rule applied to derive (looking forward) $\Gamma(\Delta)$. We only show the most interesting case of $\left(\Rightarrow^{-}\right)$, in which the proof is concluded as follows: 


$$
\frac{\Gamma\left(\neg(A \Rightarrow B),\left[A^{\prime}: \Delta, \neg B\right]\right) \quad A, \neg A^{\prime} \quad A^{\prime}, \neg A}{\Gamma\left(\neg(A \Rightarrow B),\left[A^{\prime}: \Delta\right]\right)}\left(\Rightarrow^{-}\right)
$$

We can apply the inductive hypothesis to the leftmost premise, obtaining a derivation (of no greater height) of $\Gamma\left(\neg(A \Rightarrow B),\left[A^{\prime}: \Delta, \neg B, \Sigma\right]\right)$, from which we conclude $\Gamma\left(\neg(A \Rightarrow B),\left[A^{\prime}: \Delta, \Sigma\right]\right)$ by applying (forward) the rule $\left(\Rightarrow^{-}\right)$with $A, \neg A^{\prime}$ and $A^{\prime}, \neg A$. We can also apply the inductive hypothesis to obtain a derivation of $\Gamma(\neg(A \Rightarrow$ $\left.B),\left[A^{\prime}: \Delta, \neg B\right], \Sigma\right)$ : again by applying $\left(\Rightarrow^{-}\right)$we have a derivation of $\Gamma(\neg(A \Rightarrow$ $\left.B),\left[A^{\prime}: \Delta\right], \Sigma\right)$.

We can also prove the following property, that will be useful in the subsequent proofs:

Lemma 10 Let $\Delta$ be a nested sequent and let $\Gamma()$ be a context. If $\Delta$ is derivable in $\mathcal{N} S$, then also $\Gamma(\Delta)$ is derivable in $\mathcal{N} S$.

Proof. By induction on the height of the derivation of $\Delta$. For the base cases, $\Delta$ is an instance of the axioms. We distinguish the following three subcases:

- $\Delta$ is an instance of $(A X)$, i.e. $\Delta=\Delta^{\prime}(P, \neg P)$ with $P \in A T M$ : in this case, it immediately follows that also $\Gamma(P, \neg P)$ is an instance of $(A X)$ and we are done;

- $\Delta$ is an instance of $\left(A X_{\top}\right)$, i.e. $\Delta=\Delta^{\prime}(\top)$ : in this case, also $\Gamma(\top)$ is an instance of $\left(A X_{\top}\right)$ and we are done;

- $\Delta$ is an instance of $\left(A X_{\perp}\right)$, i.e. $\Delta=\Delta^{\prime}(\neg \top)$ : reasoning exactly as we have done in the previous case, we conclude that also $\Gamma(\neg \top)$ is an instance of $\left(A X_{\perp}\right)$ and we are done.

For the inductive step, we consider all the rules of $\mathcal{N} S$ that can conclude (looking forward) the derivation of $\Delta$. To save space, we only present the most interesting case of $\left(\Rightarrow^{-}\right)$. For the other rules, we can reason in the same way, therefore the proofs are left to the reader. The derivation of $\Delta=\Delta^{\prime}\left(\neg(A \Rightarrow B),\left[A^{\prime}: \Psi\right]\right)$ is ended as follows:

$$
\frac{(1) \Delta^{\prime}\left(\neg(A \Rightarrow B),\left[A^{\prime}: \Psi, \neg B\right]\right) \quad(2) A, \neg A^{\prime} \quad(3) A^{\prime}, \neg A}{\Delta^{\prime}\left(\neg(A \Rightarrow B),\left[A^{\prime}: \Psi\right]\right)}\left(\Rightarrow^{-}\right)
$$

We can apply the inductive hypothesis to (1) to obtain a derivation of $\left(1^{\prime}\right) \Gamma(\Delta)=$ $\Gamma\left(\neg(A \Rightarrow B),\left[A^{\prime}: \Psi, \neg B\right]\right)$, from which we immediately conclude that also $\Gamma(\neg(A \Rightarrow$ $\left.B),\left[A^{\prime}: \Psi\right]\right)$ is derivable by applying $\left(\Rightarrow^{-}\right)$to $\left(1^{\prime}\right),(2)$ and $(3)$.

Lemma 11 (Invertibility) All the rules of $\mathcal{N} S$, with the exceptions of $\left(\Rightarrow^{-}\right)$and $(C E M)$, are height-preserving invertible: if $\Gamma$ has a derivation of height $h$ and it is an instance of a conclusion of a rule $(\mathbf{R})$, then also $\Gamma_{i}, i=1,2$, are derivable in $\mathcal{N} S$ with derivations of heights $h_{i} \leq h$, where $\Gamma_{i}$ are instances of the premises of $(\mathbf{R})$.

Proof. Let us first consider the rule $(I D)$. In this case, we can immediately conclude because the premise $\Gamma([A: \Delta, \neg A])$ is obtained by weakening, which is heightpreserving admissible (Lemma 9), from $\Gamma([A: \Delta])$. We proceed in the same way 
for the rule $(M P)$ : indeed, also in this case, both the premises $\Gamma(\neg(A \Rightarrow B), A)$ and $\Gamma(\neg(A \Rightarrow B), \neg B)$ are obtained by weakening from the conclusion $\Gamma(\neg(A \Rightarrow B))$ For the other rules, we proceed by induction on the height of the derivation of $\Gamma$. We only show the most interesting case of $\left(\Rightarrow^{+}\right)$, the other cases are similar and left to the reader. For the base case, consider $(*) \Gamma(A \Rightarrow B, \Delta)$ where either (i) $P \in \Delta$ and $\neg P \in \Delta$, i.e. (*) is an instance of $(A X)$, or (ii) $\top \in \Delta$, i.e. (*) is an instance of $\left(A X_{\top}\right)$, or (iii) $\neg \perp \in \Delta$, i.e. $(*)$ is an instance of $\left(A X_{\perp}\right)$; we immediately conclude that also $\Gamma([A: B], \Delta)$ is an instance of either $(A X)$ in case (i) or $\left(A X_{\top}\right)$ in case (ii) or $\left(A X_{\perp}\right)$ in case (iii).

For the inductive step, we consider each rule ending (looking forward) the derivation of $\Gamma(A \Rightarrow B)$. If the derivation is ended by an application of $\left(\Rightarrow^{+}\right)$to $\Gamma([A: B])$, we are done. Otherwise, we apply the inductive hypothesis to the premise(s) and then we conclude by applying the same rule. As an example, consider a derivation ended by an application of $\left(\Rightarrow^{-}\right)$as follows:

$$
\frac{(1) \Gamma\left(A \Rightarrow B, \neg(C \Rightarrow D),\left[C^{\prime}: \Delta, \neg D\right]\right) \quad(2) C, \neg C^{\prime} \quad(3) C^{\prime}, \neg C}{\Gamma\left(A \Rightarrow B, \neg(C \Rightarrow D),\left[C^{\prime}: \Delta\right]\right)}\left(\Rightarrow^{-}\right)
$$

We apply the inductive hypothesis to (1), obtaining a derivation of no greater height of $\left(1^{\prime}\right) \Gamma\left([A: B], \neg(C \Rightarrow D),\left[C^{\prime}: \Delta, \neg D\right]\right)$. By an application of $\left(\Rightarrow^{-}\right)$to $\left(1^{\prime}\right),(2)$, and (3) we obtain a derivation of $\Gamma\left([A: B], \neg(C \Rightarrow D),\left[C^{\prime}: \Delta\right]\right)$, and we are done. The other cases are similar and therefore omitted.

We can prove a "weak" version of invertibility also for the rules $\left(\Rightarrow^{-}\right)$and $(C E M)$. Roughly speaking, if $\Gamma\left(\neg(A \Rightarrow B),\left[A^{\prime}: \Delta\right]\right)$, which is an instance of the conclusion of $\left(\Rightarrow^{-}\right)$, is derivable, then also the sequent $\Gamma\left(\neg(A \Rightarrow B),\left[A^{\prime}: \Delta, \neg B\right]\right)$, namely the left-most premise in the rule $\left(\Rightarrow^{-}\right)$, is derivable too. Similarly for $(C E M)$, where if $\Gamma([A: \Delta],[B: \Sigma])$ is derivable, then so is the left-most premise in the rule $(C E M)$, namely $\Gamma([A: \Delta, \Sigma],[B: \Sigma])$.

Lemma 12 If $\Gamma\left(\neg(A \Rightarrow B),\left[A^{\prime}: \Delta\right]\right)$ has a derivation of height $h$ in $\mathcal{N} S$, then also $\Gamma\left(\neg(A \Rightarrow B),\left[A^{\prime}: \Delta, \neg B\right]\right)$ has a derivation of height $h^{\prime} \leq h$. If $\Gamma([A: \Delta],[B: \Sigma])$ has a derivation of height $h$ in $\mathcal{N} S$, then also $\Gamma([A: \Delta, \Sigma],[B: \Sigma])$ has a derivation of height $h^{\prime} \leq h$.

Proof. The property immediately follows since $\Gamma\left(\neg(A \Rightarrow B),\left[A^{\prime}: \Delta, \neg B\right]\right)$ (resp. $\Gamma([A: \Delta, \Sigma],[B: \Sigma]))$ is obtained by weakening from $\Gamma\left(\neg(A \Rightarrow B),\left[A^{\prime}: \Delta\right]\right)$ (resp. $\Gamma([A: \Delta],[B: \Sigma])$ ), and weakening is height-preserving admissible (Lemma 9 ).

It is worth noticing that the height preserving invertibility also preserves the number of applications of the rules in a proof, that is to say: if $\Gamma_{1}$ is derivable and it is a premise of an application of an invertible rule whose conclusion is $\Gamma_{2}$, then $\Gamma_{1}$ has a derivation containing the same rule applications as the derivation of $\Gamma_{2}$. For instance, if $\Gamma([A: \Delta])$ is derivable with a derivation $\Pi$, then $\Gamma([A: \Delta, \neg A])$ is derivable since $(I D)$ is invertible; moreover, there exists a derivation of $\Gamma([A: \Delta, \neg A])$ containing the same rules of $\Pi$, obtained by adding $\neg A$ in each $[A: \Delta]$ in the sequents of $\Pi$ from which $\Gamma([A: \Delta])$ descends. This fact will be systematically used throughout the paper, in the sense that we will assume that every proof transformation due to invertibility preserves the number of rules applications in the initial proof.

Since the rules are invertible, it follows that contraction is admissible, that is to say: 
Lemma 13 (Admissibility of contraction) Contraction is height-preserving admissible in $\mathcal{N} S$ : given a nested sequent $\Delta$, if $\Gamma(\Delta, \Delta)$ has a derivation of height $h$, then also $\Gamma(\Delta)$ has a derivation of height $h^{\prime} \leq h$. Moreover, the derivation of the contracted sequent $\Gamma(\Delta)$ does not add any rule application to the initial derivation, and we say that contractions are rule-preserving admissible.

Proof. By induction on the height of the derivation of $\Gamma(\Delta, \Delta)$. The base cases are as follows:

- $\Gamma(\Delta, \Delta)$ is an instance of $(A X)$, i.e. either $(i) \Gamma(\Delta, \Delta)=\Gamma^{\prime}(\Delta, \Delta, P, \neg P)$, or (ii) $\Delta=P$ and $\Gamma=\Gamma^{\prime}(P, P, \neg P)$, where $P \in A T M$. In case $(i)$, obviously also $\Gamma^{\prime}(\Delta, P, \neg P)$ is an instance of $(A X)$ and we are done. In case $(i i)$, we conclude similarly by observing that also $\Gamma^{\prime}(P, \neg P)$, i.e. $\Gamma(\Delta)$ is an instance of $(A X)$;

- $\Gamma(\Delta, \Delta)$ is an instance of $\left(A X_{\top}\right)$, that is to say either $\Delta=\top$ or $\Gamma(\Delta, \Delta)=$ $\Gamma^{\prime}(\Delta, \Delta, \top)$. In both cases, obviously also $\Gamma(\Delta)$ is an instance of $\left(A X_{\top}\right)$ and we are done;

- $\Gamma(\Delta, \Delta)$ is an instance of $\left(A X_{\perp}\right)$, that is to say either $\Delta=\neg \perp$ or $\Gamma(\Delta, \Delta)=$ $\Gamma^{\prime}(\Delta, \Delta, \neg \perp)$. Again, in both cases also $\Gamma(\Delta)$ is an instance of $\left(A X_{\perp}\right)$.

For the inductive step, we distinguish two cases:

- $\Delta$ is not principal in the application of the rule ending (looking forward) the derivation of $\Gamma(\Delta, \Delta)$. In all these cases, the two instances of $\Delta$ also belong to the premise(s) of the rule, to which we can apply the inductive hypothesis and then conclude by an application of the same rule. As an example, consider a derivation ended by an application of $(C E M)$ as follows:

$$
\frac{(1) \Gamma(\Delta, \Delta,[A: \Psi, \Sigma],[B: \Sigma]) \quad A, \neg B \quad B, \neg A}{\Gamma(\Delta, \Delta,[A: \Psi],[B: \Sigma])}(C E M)
$$

We can apply the inductive hypothesis on (1) to obtain a derivation (of no greater height) of $\left(1^{\prime}\right) \Gamma(\Delta,[A: \Psi],[B: \Sigma])$, then we conclude by an application of $(C E M)$ as follows:

$$
\frac{\left(1^{\prime}\right) \Gamma(\Delta,[A: \Psi, \Sigma],[B: \Sigma]) \quad A, \neg B \quad B, \neg A}{\Gamma(\Delta,[A: \Psi],[B: \Sigma])}(C E M)
$$

- $\Delta$ is principal in the application of the rule ending (forward) the derivation of $\Gamma(\Delta, \Delta)$. We proceed by induction on the cardinality $|\Delta|$ of $\Delta$, defined in the usual way: if $\Delta=F_{1}, F_{2}, \ldots, F_{n}$, where $F_{i}$ is either a formula or a context $[A: \Psi]$, then $|\Delta|=n$. For the base case, $|\Delta|=1$, that is to say $\Delta=F$ where $F$ is either a formula or a context $[A: \Psi]$. We further distinguish between two groups of rules:

- $\left(\Rightarrow^{-}\right),(I D),(M P),(C E M)$ : in these rules, the principal formula is copied into one of the premises, therefore we apply the inductive hypothesis to such premise and conclude by applying the same rule. Let us first consider the case of $\left(\Rightarrow^{-}\right)$and a derivation ending as follows:

$$
\frac{(1) \Gamma\left(\neg(A \Rightarrow B), \neg(A \Rightarrow B),\left[A^{\prime}: \Psi, \neg B\right]\right) \quad(2) A, \neg A^{\prime} \quad(3) A^{\prime}, \neg A}{\Gamma\left(\neg(A \Rightarrow B), \neg(A \Rightarrow B),\left[A^{\prime}: \Psi\right]\right)}\left(\Rightarrow^{-}\right)
$$


We can apply the inductive hypothesis to (1), obtaining a derivation (of no greater height $)$ of $\Gamma\left(\neg(A \Rightarrow B),\left[A^{\prime}: \Psi, \neg B\right]\right)$. Together with (2) and (3), we conclude with an application of $\left(\Rightarrow^{-}\right)$.

If the derivation is ended as follows:

$$
\frac{(1) \Gamma\left(\neg(A \Rightarrow B),\left[A^{\prime}: \Psi, \neg B\right],\left[A^{\prime}: \Psi\right]\right) \quad(2) A, \neg A^{\prime} \quad(3) A^{\prime}, \neg A}{\Gamma\left(\neg(A \Rightarrow B),\left[A^{\prime}: \Psi\right],\left[A^{\prime}: \Psi\right]\right)}\left(\Rightarrow^{-}\right)
$$

we observe that also $\left(1^{\prime}\right) \Gamma\left(\neg(A \Rightarrow B),\left[A^{\prime}: \Psi, \neg B\right],\left[A^{\prime}: \Psi, \neg B\right]\right)$ has a derivation of no greater height than (1) since weakening is admissible (Lemma 9), to which we can apply the inductive hypothesis to obtain a derivation of $\left(1^{\prime \prime}\right) \Gamma\left(\neg(A \Rightarrow B),\left[A^{\prime}: \Psi, \neg B\right]\right)$. We conclude from $\left(1^{\prime \prime}\right)$, (2) and (3) with an application of $\left(\Rightarrow^{-}\right)$.

Concerning $(I D)$, we have a derivation ending as follows:

$$
\frac{(1) \Gamma([A: \Psi, \neg A],[A: \Psi])}{\Gamma([A: \Psi],[A: \Psi])}(I D)
$$

By Lemma 9, from (1) we have a derivation of no greater height of $\left(1^{\prime}\right) \Gamma([A: \Psi, \neg A],[A: \Psi, \neg A])$, to which we apply the inductive hypothesis to obtain a derivation of no greater height of $\Gamma([A: \Psi, \neg A])$, from which we conclude by an application of $(I D)$.

Let us now consider $(M P)$. The derivation is ended as follows:

$$
\frac{(1) \Gamma(\neg(A \Rightarrow B), \neg(A \Rightarrow B), A) \quad(2) \Gamma(\neg(A \Rightarrow B), \neg(A \Rightarrow B), \neg B)}{\Gamma(\neg(A \Rightarrow B), \neg(A \Rightarrow B))}(M P)
$$

We can apply the inductive hypothesis to (1), obtaining a derivation of $\left(1^{\prime}\right) \Gamma(\neg(A \Rightarrow B), A)$, and to $(2)$, obtaining a derivation of $\left(2^{\prime}\right) \Gamma(\neg(A \Rightarrow$ $B), \neg B)$, then we can conclude by an application of $(M P)$ to $\left(1^{\prime}\right)$ and $\left(2^{\prime}\right)$ For $(C E M)$ we proceed similarly to what done for $\left(\Rightarrow^{-}\right)$. If the derivation ends as follows:

$$
\frac{(1) \Gamma([A: \Psi, \Sigma],[B: \Sigma],[B: \Sigma]) \quad(2) A, \neg B \quad(3) B, \neg A}{\Gamma([A: \Psi],[B: \Sigma],[B: \Sigma])}(C E M)
$$

we apply the inductive hypothesis to $(1)$, obtaining a derivation of $\Gamma([A$ : $\Psi, \Sigma],[B: \Sigma])$, then we conclude by applying $(C E M)$ together with $(2)$ and (3). If the derivation ends as follows:

$$
\frac{(1) \Gamma([A: \Psi, \Sigma],[A: \Psi],[B: \Sigma]) \quad(2) A, \neg B \quad(3) B, \neg A}{\Gamma([A: \Psi],[A: \Psi],[B: \Sigma])}(C E M)
$$

we first observe that also $\left(1^{\prime}\right) \Gamma([A: \Psi, \Sigma],[A: \Psi, \Sigma],[B: \Sigma])$ is derivable (with a derivation of no greater height than (1)) by Lemma 9. We apply the inductive hypothesis on $\left(1^{\prime}\right)$ to obtain a derivation of $\left(1^{\prime \prime}\right) \Gamma([A: \Psi, \Sigma],[B: \Sigma])$. We conclude by an application of $(C E M)$ to $\left(1^{\prime \prime}\right),(2)$ and $(3)$.

- $\left(\Rightarrow^{+}\right),(\neg),\left(\wedge^{+}\right),\left(\wedge^{-}\right),\left(\vee^{+}\right),\left(\vee^{-}\right),\left(\rightarrow^{+}\right),\left(\rightarrow^{-}\right)$: in these rules, the principal formula is not copied into the premise(s), therefore we cannot 
apply the inductive hypothesis. However, since all these rules are heightpreserving invertible (Lemma 11), we apply the inductive hypothesis to the premise of a (further) application of the same rule to the premise, as in the example of $\left(\Rightarrow^{+}\right)$when the derivation ends as follows (the other cases are similar and left to the reader):

$$
\frac{(1) \Gamma([A: B], A \Rightarrow B)}{\Gamma(A \Rightarrow B, A \Rightarrow B)}\left(\Rightarrow^{+}\right)
$$

By Lemma 11, we have a derivation of no greater height than (1) of $\Gamma([A: B],[A: B])$, to which we can apply the inductive hypothesis to obtain a derivation of $\Gamma([A: B])$, from which we conclude by an application of $\left(\Rightarrow^{+}\right)$.

For the inductive step, we have that $|\Delta|=n>1$, that is to say $\Delta=\Delta^{\prime}, F$. Therefore, we have a derivation of $\Gamma\left(\Delta^{\prime}, \Delta^{\prime}, F, F\right)$. We have that $\left|\Delta^{\prime}\right|<n$, as well as $|F|<n$, therefore we can apply the inductive hypothesis two times to obtain a derivation of $\Gamma\left(\Delta^{\prime}, F\right)=\Gamma(\Delta)$ and we are done.

\subsection{Soundness of the calculi $\mathcal{N} S$}

To improve readability, we slightly abuse the notation identifying a sequent $\Gamma$ with its interpreting formula $\mathcal{F}(\Gamma)$, thus we shall write $A \Rightarrow \Delta, \Gamma \wedge \Delta$, etc. instead of $A \Rightarrow \mathcal{F}(\Gamma), \mathcal{F}(\Gamma) \wedge \mathcal{F}(\Delta)$. First of all we prove that nested inference is sound. The proof is inspired to the one of Lemma 2.8 in [7].

Lemma 14 Let $\Gamma()$ be any context. If the formula $A_{1} \wedge \ldots \wedge A_{n} \rightarrow B$, with $n \geq 0$, is $(C K\{+X\})$-valid, then also $\Gamma\left(A_{1}\right) \wedge \ldots \wedge \Gamma\left(A_{n}\right) \rightarrow \Gamma(B)$ is $(C K\{+X\})$ valid.

Proof. By induction on the depth of a context $\Gamma()$. Let $d(\Gamma())=0$, then $\Gamma=\Lambda,()$. Since $A_{1} \wedge \ldots \wedge A_{n} \rightarrow B$ is valid, by propositional reasoning, we have that also $\left(\Lambda \vee A_{1}\right) \wedge \ldots\left(\Lambda \vee A_{n}\right) \rightarrow(\Lambda \vee B)$ is valid, that is $\Gamma\left(A_{1}\right) \wedge \ldots \wedge \Gamma\left(A_{n}\right) \rightarrow \Gamma(B)$ is valid. Let $d(\Gamma())>0$, then $\Gamma()=\Delta,[C: \Sigma()]$. By inductive hypothesis, we have that $\Sigma\left(A_{1}\right) \wedge \ldots \wedge \Sigma\left(A_{n}\right) \rightarrow \Sigma(B)$ is valid. By (RCK), we obtain that also $\left(C \Rightarrow \Sigma\left(A_{1}\right)\right) \wedge \ldots \wedge\left(C \Rightarrow \Sigma\left(A_{n}\right)\right) \rightarrow(C \Rightarrow \Sigma(B))$ is valid. Then, we get that $\left(\Lambda \vee\left(C \Rightarrow \Sigma\left(A_{1}\right)\right)\right) \wedge \ldots \wedge\left(\Lambda \vee\left(C \Rightarrow \Sigma\left(A_{n}\right)\right)\right) \rightarrow(\Lambda \vee(C \Rightarrow \Sigma(B)))$ is also valid, that is $\Gamma\left(A_{1}\right) \wedge \ldots \wedge \Gamma\left(A_{n}\right) \rightarrow \Gamma(B)$ is valid.

Theorem 15 (Soundness of $\mathcal{N} S$ ) If $\Gamma$ is derivable in $\mathcal{N} S$, then $\Gamma$ is valid.

Proof. By induction on the height of the derivation of $\Gamma$. If $\Gamma$ is an axiom, that is $\Gamma=\Gamma(P, \neg P)$, then trivially $P \vee \neg P$ is valid; by Lemma 14 (case $n=0$ ), we get $\Gamma(P, \neg P)$ is valid. Similarly for $\Gamma(\top)$ and $\Gamma(\neg \perp)$. Otherwise $\Gamma$ is obtained by a rule $(\mathbf{R})$ :

- $(\mathbf{R})$ is a propositional rule, say

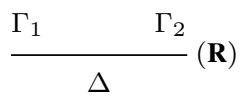


We first prove that $\Gamma_{1} \wedge \Gamma_{2} \rightarrow \Delta$ is valid. All rules are easy, since for the empty context they are nothing else than trivial propositional tautologies. We can then use Lemma 14 to propagate them to any context. For instance, let the rule $(\mathbf{R})$ be $\left(\vee^{-}\right)$. Then $(\neg A \wedge \neg B) \rightarrow \neg(A \vee B)$ and, by the previous Lemma 14, we get that $\Gamma(\neg A) \wedge \Gamma(\neg B) \rightarrow \Gamma(\neg(A \vee B))$. Thus if $\Gamma$ is derived by $(\mathbf{R})$ from $\Gamma_{1}, \Gamma_{2}$, we use the inductive hypothesis that $\Gamma_{1}$ and $\Gamma_{2}$ are valid and the above fact to conclude.

- $(\mathbf{R})$ is $\left(\Rightarrow^{+}\right)$: trivial by inductive hypothesis.

- $(\mathbf{R})$ is $\left(\Rightarrow^{-}\right)$then $\Gamma=\Gamma\left(\neg(A \Rightarrow B),\left[A^{\prime}: \Delta\right]\right)$ is derived from $(i) \Gamma(\neg(A \Rightarrow$ $\left.B),\left[A^{\prime}: \Delta, \neg B\right]\right),(i i) \neg A, A^{\prime},(i i i) \neg A^{\prime}, A$. By inductive hypothesis we have that $A \leftrightarrow A^{\prime}$ is valid. We show that also $(*)\left[\neg(A \Rightarrow B) \vee\left(A^{\prime} \Rightarrow(\Delta \vee \neg B)\right)\right] \rightarrow$ $\left[\neg(A \Rightarrow B) \vee\left(A^{\prime} \Rightarrow \Delta\right)\right]$ is valid, then we apply Lemma 14 and the inductive hypothesis to conclude. To prove $(*)$, by $(\mathrm{RCK})$ we have that the following is valid: $\left[\left(A^{\prime} \Rightarrow B\right) \wedge\left(A^{\prime} \Rightarrow(\Delta \vee \neg B)\right)\right] \rightarrow\left(A^{\prime} \Rightarrow \Delta\right)$. Since $A \leftrightarrow A^{\prime}$ is valid, by (RCEA) we get that $(A \Rightarrow B) \rightarrow\left(A^{\prime} \Rightarrow B\right)$ is valid, so that also $(A \Rightarrow B) \rightarrow\left(\left(A^{\prime} \Rightarrow(\Delta \vee \neg B)\right) \rightarrow\left(A^{\prime} \Rightarrow \Delta\right)\right)$ is valid, then we conclude by propositional reasoning.

- $(\mathbf{R})$ is $(I D)$, then $\Gamma=\Gamma([A: \Delta])$ is derived from $\Gamma([A: \Delta, \neg A])$. We show that $(A \Rightarrow(\Delta \vee \neg A)) \rightarrow(A \Rightarrow \Delta)$ is valid in $\mathrm{CK}+\mathrm{ID}$, then we conclude by Lemma 14 and by the inductive hypothesis. The mentioned formula is derivable: by $(\mathrm{RCK})$ we obtain $(A \Rightarrow A) \rightarrow((A \Rightarrow(\Delta \vee \neg A)) \rightarrow(A \Rightarrow \Delta))$ so that we conclude by $(I D)$.

- $(\mathbf{R})$ is $(M P)$, then $\Gamma=\Gamma(\neg(A \Rightarrow B))$ is derived from $(i) \Gamma(\neg(A \Rightarrow B), A)$ and $($ ii $) \Gamma(\neg(A \Rightarrow B), \neg B)$. We show that $(*)(\neg(A \Rightarrow B) \wedge A) \wedge(\neg(A \Rightarrow$ $B) \wedge \neg B) \rightarrow(\neg(A \Rightarrow B))$ is valid in CK+MP, then we conclude by Lemma 14 and by the inductive hypothesis. By Axiom MP we have $(A \Rightarrow B) \rightarrow$ $\neg(A \wedge \neg B)$ is valid. Thus also $(A \Rightarrow B) \rightarrow((A \Rightarrow B) \wedge \neg(A \wedge \neg B))$ is valid, whence by contraposition $\neg((A \Rightarrow B) \wedge \neg(A \wedge \neg B)) \rightarrow \neg(A \Rightarrow B)$ and $(\neg(A \Rightarrow B) \vee(A \wedge \neg B)) \rightarrow \neg(A \Rightarrow B)$, we get (*) by immediate propositional reasoning.

- $(\mathbf{R})$ is $(C E M)$, thus $\Gamma=\Gamma\left([A: \Delta],\left[A^{\prime}, \Sigma\right]\right)$ and it is derived from $(i) \Gamma([A$ : $\left.\Delta, \Sigma],\left[A^{\prime}: \Sigma\right]\right),(i i) \neg A, A^{\prime},($ iii $) \neg A^{\prime}, A$. By inductive hypothesis $A \leftrightarrow A^{\prime}$ is valid. We first show that $(* *)(A \Rightarrow(\Delta \vee \Sigma)) \rightarrow\left((A \Rightarrow \Delta) \vee\left(A^{\prime} \Rightarrow \Sigma\right)\right)$. Then we conclude as before by Lemma 14 and inductive hypothesis. To prove (**), we notice that the following is derivable by (RCK): $(A \Rightarrow(\Delta \vee \Sigma)) \rightarrow[(A \Rightarrow$ $\neg \Delta) \rightarrow(A \Rightarrow \Sigma)]$. By $(C E M)$, the following is valid: $(A \Rightarrow \Delta) \vee(A \Rightarrow \neg \Delta)$. Thus we get that $(A \Rightarrow(\Delta \vee \Sigma)) \rightarrow[(A \Rightarrow \Delta) \vee(A \Rightarrow \Sigma)]$ is valid. Since $A \leftrightarrow A^{\prime}$ is valid, (by RCEA) we have that also $(A \Rightarrow \Sigma) \rightarrow\left(A^{\prime} \Rightarrow \Sigma\right)$ is valid, obtaining (**). 


\subsection{Completeness of the calculi $\mathcal{N} S$}

Completeness is an easy consequence of the admissibility of the following rule cut:

$$
\frac{\Gamma(F) \quad \Gamma(\neg F)}{\Gamma(\emptyset)}(c u t)
$$

where $F$ is a formula. The standard proof of admissibility of cut proceeds by a double induction over the complexity of $F$ and the sum of the heights of the derivations of the two premises of $(c u t)$, in the sense that we replace one cut by one or several cuts on formulas of smaller complexity, or on sequents derived by shorter derivations.

However, in $\mathcal{N} S$ the standard proof does not work in the following case, in which the cut formula $F$ is a conditional formula $A \Rightarrow B$ :

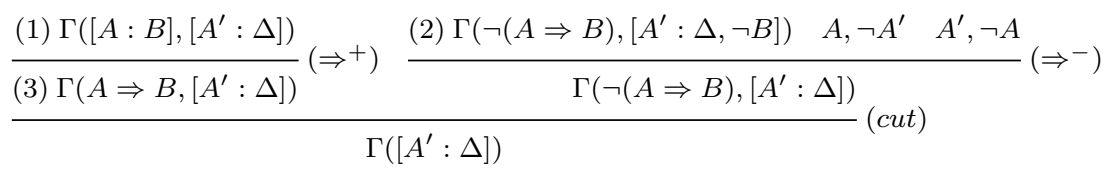

Indeed, even if we apply the inductive hypothesis on the heights of the derivations of the premises to cut (2) and (3), obtaining (modulo weakening, which is admissible by Lemma 9) a derivation of

$$
\left(2^{\prime}\right) \Gamma\left(\left[A^{\prime}: \Delta, \neg B\right],\left[A^{\prime}: \Delta\right]\right),
$$

we cannot apply the inductive hypothesis on the complexity of the cut formula to $\left(2^{\prime}\right)$ and

$$
\left(1^{\prime}\right) \Gamma\left([A: \Delta, B],\left[A^{\prime}: \Delta\right]\right)
$$

(obtained from (1) again by weakening). Such an application would be needed in order to obtain a derivation of $\Gamma\left(\left[A^{\prime}: \Delta\right],\left[A^{\prime}: \Delta\right]\right)$ and then to conclude $\Gamma\left(\left[A^{\prime}: \Delta\right]\right)$ since contraction is admissible (Lemma 13): indeed, the two contexts are different, we have $\left[A^{\prime}: \Delta, \neg B\right]$ in $\left(2^{\prime}\right)$ whereas we have $[A: \Delta, B]$ in $\left(1^{\prime}\right)$.

In order to tackle this problem, we need to prove another property, namely that if $A$ and $A^{\prime}$ are equivalent, if a sequent $\Gamma([A: \Delta])$ is derivable in $\mathcal{N} S$, then also $\Gamma\left(\left[A^{\prime}: \Delta\right]\right)$, obtained by replacing $A$ with the equivalent formula $A^{\prime}$, is also derivable. In turn, we need (cut) to prove this property, therefore we prove both the properties (admissibility of (cut) and "substitution" of $A$ with $A^{\prime}$ ) by mutual induction. We first consider the calculi without MP.

\subsubsection{Admissibility of cut for systems without MP}

In order to prove the admissibility of cut for $\mathcal{N} S$, we proceed as follows. First, we show that if $A, \neg A^{\prime}$ and $A^{\prime}, \neg A$ are derivable, then if $\Gamma([A: \Delta])$ is derivable, then $\Gamma\left(\left[A^{\prime}: \Delta\right]\right)$, obtained by replacing $[A: \Delta]$ with $\left[A^{\prime}: \Delta\right]$, is also derivable. We prove that cut is admissible by "splitting" the notion of cut in two propositions:

Theorem 16 In $\mathcal{N} S$, the following propositions hold:

- (A) If $\Gamma(F)$ and $\Gamma(\neg F)$ are derivable, so is $\Gamma(\emptyset)$, i.e. (cut) is admissible in $\mathcal{N} S$; 
- (B) if (I) $\Gamma([A: \Delta]),(I I) A, \neg A^{\prime}$ and (III) $A^{\prime}, \neg A$ are derivable, then $\Gamma\left(\left[A^{\prime}: \Delta\right]\right)$ is derivable.

Proof. To make the structure of the induction clear call: $C u t(c, h)$ the property (A) for any $\Gamma$ and any formula $F$ of complexity $c$ and such that the sum of the heights of derivation of the premises is $h$. Similarly call $S u b(c)$ the assertion that (B) holds for any $\Gamma$ and any formula $A$ of complexity $c$. The proof of both (A) and (B) is by mutual induction on the complexity $c$. We show the following facts:

$$
\begin{aligned}
& \text { (A) } \forall c \forall h\left(\left(\forall c^{\prime}<c \forall h^{\prime} C u t\left(c^{\prime}, h^{\prime}\right) \wedge \forall h^{\prime \prime}<h C u t\left(c, h^{\prime \prime}\right)\right) \rightarrow C u t(c, h)\right) \\
& \text { (B) } \forall c(\forall h C u t(c, h) \rightarrow \operatorname{Sub}(c))
\end{aligned}
$$

This will prove that $\forall c \forall h C u t(c, h)$ and $\forall c S u b(c)$. The proof of (B) (that is that $S u b(c)$ holds) in itself is by induction on the height $h$ of the derivation of the premise (I) of (B).

Let us first consider (A). We have the following cases:

- (at least) one of the premises of (cut) is an instance of the axioms. Suppose that the left premise is an instance of $(A X)$. In case it has the form $\Gamma(P, \neg P, F)$, then also $\Gamma(P, \neg P)$ is an instance of $(A X)$ and we are done. Otherwise, we have $\Gamma(F, \neg F)$. In this case, the right premise of (cut) has the form $\Gamma(\neg F, \neg F)$, whereas the conclusion is $\Gamma(\neg F)$ : since contraction is admissible (Lemma 13), we conclude that $\Gamma(\neg F)$ is derivable and we are done. The other cases are symmetric. The cases in which one premise of $(c u t)$ is an instance of either $\left(A X_{\top}\right)$ or $\left(A X_{\perp}\right)$ are easy and left to the reader;

- the last step of one of the two premises is obtained by a rule $(\mathbf{R})$ in which $F$ is $n o t$ the principal formula. This case is standard, we can permute $(\mathbf{R})$ over the cut, i.e. we cut the premise(s) of (R) and then we apply (R) to the result of cut. As an example, we present the case of $\left(\Rightarrow^{-}\right)$:

$$
\begin{aligned}
& \text { (1) } \Gamma\left(\neg(A \Rightarrow B),\left[A^{\prime}: \Delta, \neg B\right], F\right) \\
& \text { (2) } A, \neg A^{\prime} \\
& \text { (3) } A^{\prime}, \neg A
\end{aligned}
$$

Since weakening is height-preserving admissible (Lemma 9), from (4) we obtain a derivation of no greater height of $\left(4^{\prime}\right) \Gamma\left(\neg(A \Rightarrow B),\left[A^{\prime}: \Delta, \neg B\right], \neg F\right)$. We then apply the inductive hypothesis on the height to cut (1) and $\left(4^{\prime}\right)$, obtaining a derivation of $(5) \Gamma\left(\neg(A \Rightarrow B),\left[A^{\prime}: \Delta, \neg B\right]\right)$; we conclude by an application of $\left(\Rightarrow^{-}\right)$to $(5),(2)$, and (3);

- $F$ is the principal formula in the last step of both derivations of the premises of the cut inference. There are seven subcases: $F$ is introduced (a) by $\left(\wedge^{-}\right)-\left(\wedge^{+}\right)$, (b) by $\left(\mathrm{V}^{-}\right)-\left(\mathrm{V}^{+}\right)$, (c) by $\left(\rightarrow^{-}\right)-\left(\rightarrow^{+}\right)$, (d) by $\left(\Rightarrow^{-}\right)-\left(\Rightarrow^{+}\right)$, (e) by $\left(\Rightarrow^{-}\right)$$(I D)$, (f) by $\left(\Rightarrow^{-}\right)-(C E M)$, (g) by $(C E M)$ - (ID). The list is exhaustive. Let us consider each case.

For case a), the derivation is ended as follows: 


$$
\frac{\frac{(1) \Gamma(\neg A, \neg B)}{\Gamma(\neg(A \wedge B))}\left(\wedge^{-}\right) \frac{(2) \Gamma(A) \quad(3) \Gamma(B)}{\Gamma(A \wedge B)}(\text { cut })}{\Gamma(\emptyset)}
$$

First of all, since weakening is admissible (Lemma 9), from (2) we obtain a derivation of $\left(2^{\prime}\right) \Gamma(A, \neg B)$. We can then apply the inductive hypothesis for statement (A) on the complexity of the cut formula to $(1)$ and $\left(2^{\prime}\right)$ to obtain a derivation of $(4) \Gamma(\neg B)$. We conclude by applying again the inductive hypothesis for statement (A) on the complexity of the cut formula to cut (4) and (3).

The cases b) and c) are analogous and therefore left to the reader.

For case d), the derivation is as follows:

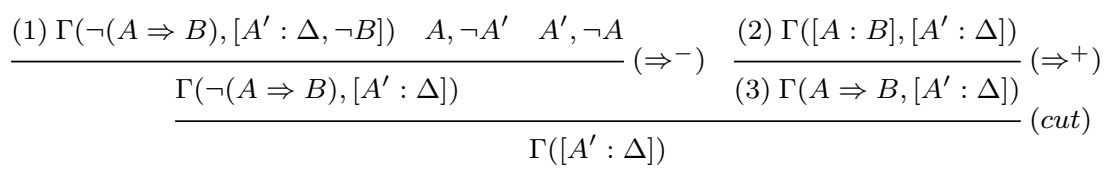

First of all, since we have proofs for $A, \neg A^{\prime}$ and for $A^{\prime}, \neg A$ and $c p(A)<$ $c p(A \Rightarrow B)$, we can apply the inductive hypothesis for (B) to (2), obtaining a derivation of $\left(2^{\prime}\right) \Gamma\left(\left[A^{\prime}: B\right],\left[A^{\prime}: \Delta\right]\right)$. By Lemma 9 , from $(3)$ we obtain a derivation of at most the same height of $\left(3^{\prime}\right) \Gamma\left(A \Rightarrow B,\left[A^{\prime}: \Delta, \neg B\right]\right)$. We can then conclude as follows: we first apply the inductive hypothesis on the height for (A) to cut (1) and $\left(3^{\prime}\right)$, obtaining a derivation of (4) $\Gamma\left(\left[A^{\prime}: \Delta, \neg B\right]\right)$. By Lemma 9, we have also a derivation of $\left(4^{\prime}\right) \Gamma\left(\left[A^{\prime}: \Delta, \neg B\right],\left[A^{\prime}: \Delta\right]\right)$. Again by Lemma 9 , from $\left(2^{\prime}\right)$ we obtain a derivation of $\left(2^{\prime \prime}\right) \Gamma\left(\left[A^{\prime}: \Delta, B\right],\left[A^{\prime}: \Delta\right]\right)$. We then apply the inductive hypothesis on the complexity of the cut formula to cut $\left(2^{\prime \prime}\right)$ and $\left(4^{\prime}\right)$, obtaining a derivation of $\Gamma\left(\left[A^{\prime}: \Delta\right],\left[A^{\prime}: \Delta\right]\right)$, from which we conclude since contraction is admissible (Lemma 13).

For case (e), the derivation is as follows:

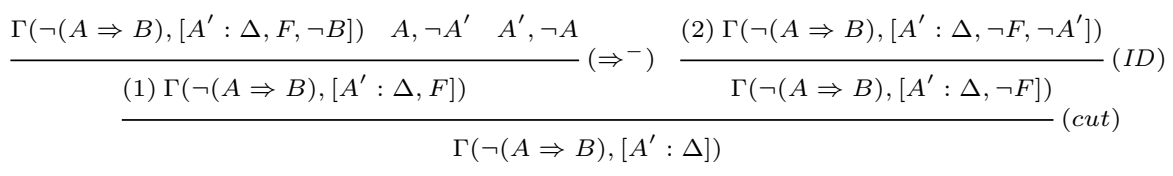

Since weakening is height-preserving admissible (Lemma 9), from (1) we obtain a derivation, of at most the same height, of $\left(1^{\prime}\right) \Gamma\left(\neg(A \Rightarrow B),\left[A^{\prime}: \Delta, F, \neg A^{\prime}\right]\right)$; we conclude as follows, by applying the inductive hypothesis for $(\mathrm{A})$ on the height:

$$
\frac{\left(1^{\prime}\right) \Gamma\left(\neg(A \Rightarrow B),\left[A^{\prime}: \Delta, F, \neg A^{\prime}\right]\right) \quad(2) \Gamma\left(\neg(A \Rightarrow B),\left[A^{\prime}: \Delta, \neg F, \neg A^{\prime}\right]\right)}{\frac{\Gamma\left(\neg(A \Rightarrow B),\left[A^{\prime}: \Delta, \neg A^{\prime}\right]\right)}{\Gamma\left(\neg(A \Rightarrow B),\left[A^{\prime}: \Delta\right]\right)}(I D)}(\text { cut })
$$


For case (f), the derivation is as follows:

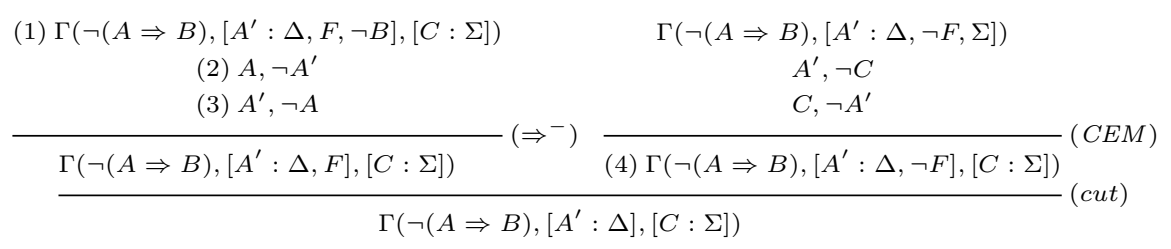

By Lemma 9, from (4) we obtain a derivation of at most the same height for $\left(4^{\prime}\right) \Gamma\left(\neg(A \Rightarrow B),\left[A^{\prime}: \Delta, \neg F, \neg B\right],[C: \Sigma]\right)$, then we can conclude as follows by applying the inductive hypothesis for (A) on the height:

$$
\begin{aligned}
& \text { (1) } \Gamma\left(\neg(A \Rightarrow B),\left[A^{\prime}: \Delta, F, \neg B\right],[C: \Sigma]\right) \\
& \left.\frac{\left(4^{\prime}\right) \Gamma\left(\neg(A \Rightarrow B),\left[A^{\prime}: \Delta, \neg F, \neg B\right],[C: \Sigma]\right)}{\frac{\Gamma\left(\neg(A \Rightarrow B),\left[A^{\prime}: \Delta, \neg B\right],[C: \Sigma]\right)}{\Gamma(\neg(A \Rightarrow)}(2) A, \neg A^{\prime} \quad(3) A^{\prime}, \neg A} \quad\left(A^{\prime}: \Delta\right],[C: \Sigma]\right)
\end{aligned}
$$

For case $(\mathrm{g})$, the derivation is as follows:

$$
\frac{(1) \Gamma([A: \Delta, F, \neg A],[B: \Sigma])}{\Gamma([A: \Delta, F],[B: \Sigma])}(I D) \frac{\Gamma([A: \Delta, \neg F, \Sigma]) \quad A, \neg B \quad B, \neg A}{(2) \Gamma([A: \Delta, \neg F],[B: \Sigma])}(C E M)
$$

By Lemma 9, from (2) we obtain a derivation of at most the same height of $\left(2^{\prime}\right) \Gamma([A: \Delta, \neg F, \neg A],[B: \Sigma])$. We can then apply the inductive hypothesis on the height to cut $(1)$ and $\left(2^{\prime}\right)$ to obtain $\Gamma([A: \Delta, \neg A],[B: \Sigma])$, from which we conclude by an application of $(I D)$.

Concerning (B), we proceed by induction on the height $h$ of the premise (I), as follows:

- Base case: $\Gamma([A: \Delta])$ is an instance of the axioms, that is to say either $\Delta=$ $\Lambda(P, \neg P)$ or $\Delta=\Lambda(\top)$ or $\Delta=\Lambda(\neg \perp)$ : we immediately conclude that also $\Gamma\left(\left[A^{\prime}: \Delta\right]\right)$ is an instance of the axioms, and we are done;

- Inductive step: we have to consider all possible rules ending (looking forward) the derivation of $\Gamma([A: \Delta])$. We only show the most interesting case, when $\left(\Rightarrow^{-}\right)$is applied by using $[A: \Delta]$ as principal formula. The derivation ends as follows:

$$
\frac{(1) \Gamma(\neg(C \Rightarrow D),[A: \Delta, \neg D]) \quad(2) C, \neg A \quad(3) A, \neg C}{\Gamma(\neg(C \Rightarrow D),[A: \Delta])}\left(\Rightarrow^{-}\right)
$$

We can apply the inductive hypothesis to (1) to obtain a derivation of $\left(1^{\prime}\right) \Gamma(\neg(C \Rightarrow$ $\left.D),\left[A^{\prime}: \Delta, \neg D\right]\right)$. Since weakening is admissible (Lemma 9), from $(I I)$ we obtain a derivation of $\left(I I^{\prime}\right) C, A, \neg A^{\prime}$, from $(I I I)$ we obtain a derivation of $\left(I I I^{\prime}\right) A^{\prime}, \neg A, \neg C$. Again by weakening, from (2) and (3) we obtain derivations of $\left(2^{\prime}\right) C, \neg A, \neg A^{\prime}$ and $\left(3^{\prime}\right) A^{\prime}, A, \neg C$, respectively. We apply the inductive hypothesis of (A) that is that cut holds for the formula $A$ (of a given complexity c) and conclude as follows:

$$
\begin{aligned}
& \left(I I^{\prime}\right) C, A, \neg A^{\prime} \quad\left(I I I^{\prime}\right) A^{\prime}, \neg A, \neg C \\
& \frac{\left(1^{\prime}\right) \Gamma\left(\neg(C \Rightarrow D),\left[A^{\prime}: \Delta, \neg D\right]\right) \frac{\left(2^{\prime}\right) C, \neg A, \neg A^{\prime}}{C, \neg A^{\prime}}(\text { cut }) \frac{\left(3^{\prime}\right) A^{\prime}, A, \neg C}{A^{\prime}, \neg C}\left(\Rightarrow^{-}\right)}{\Gamma\left(\neg(C \Rightarrow D),\left[A^{\prime}: \Delta\right]\right)}(\text { cut })
\end{aligned}
$$


Let us now consider the case of the calculi for systems allowing the axiom MP.

\subsubsection{Admissibility of cut for systems CK+MP and CK+ID+MP}

The proof is slightly different from the one of Theorem 16 above, that is why we prove it in a separate theorem. To make cut elimination work, we need to show the admissibility of the following rule:

$$
\frac{\Gamma(A) \Gamma([A: \Delta])}{\Gamma(\Delta)}
$$

This rule corresponds to a generalization of the MP axiom and it is needed to handle the elimination of a cut in which one of the premises is a negative conditional $\neg(A \Rightarrow B)$ introduced by an instance of the MP rule.

Theorem 17 In systems $C K+M P$ and $C K+I D+M P$ the following propositions hold:

(a) If $\Gamma(F)$ and $\Gamma(\neg F)$ are derivable, then so is $\Gamma(\emptyset)$, i.e. (cut) is admissible in $\mathcal{N} S$

(b) if (I) $\Gamma([A: \Delta]),(I I) \neg A, A^{\prime}$, and $(I I I) \neg A^{\prime}, A$ are derivable, then so is $\Gamma\left(\left[A^{\prime}: \Delta\right]\right)$;

(c) if $\Gamma([A: \Delta])$ and $\Gamma(A)$ is derivable, then so is $\Gamma(\Delta)$.

Proof. To make the structure of the induction clear call: $C u t(c, h)$ the property (a) for any $\Gamma$ and any formula $A$ of complexity $c$ and such that the sum of the heights of derivation of the premises is $h, S u b(c)$ the assertion that (b) holds for any $\Gamma$ and any formula $A$ of complexity $c$ and, finally, $M P(c)$ the assertion that (c) holds for any $\Gamma, \Delta$ and any formula $A$ of complexity $c$. The proof of the three statements is by mutual induction on the complexity $c$. We show the following facts:

(a) $\forall c \forall h\left(\left(\forall c^{\prime}<c \forall h^{\prime} C u t\left(c^{\prime}, h^{\prime}\right) \wedge \forall h^{\prime \prime}<h C u t\left(c, h^{\prime \prime}\right)\right) \rightarrow C u t(c, h)\right)$

(b) $\forall c(\forall h C u t(c, h) \rightarrow S u b(c))$

(c) $\forall c(\forall h C u t(c, h) \rightarrow M P(c))$.

This will prove that $\forall c \forall h C u t(c, h), \forall c S u b(c)$ and $\forall c M P(c)$. The proof of (a) is the same as the one of (A) of Theorem 16 except in the case the principal formula $\neg(C \Rightarrow D)$ is introduced by the $(M P)$ rule, namely we have the following:

$$
\frac{(1) \Gamma(\neg(C \Rightarrow D), C) \quad(2) \Gamma(\neg(C \Rightarrow D), \neg D)}{\Gamma(\neg(C \Rightarrow D))}(M P) \frac{(3) \Gamma([C: D])}{(4) \Gamma(C \Rightarrow D)}(\Rightarrow R)
$$

In this case we proceed as follows: first of all, since weakening is height-preserving admissibile (Lemma 9), from (4) we obtain a derivation of no greater height of 
$\left(4^{\prime}\right) \Gamma(C \Rightarrow D, C)$ and of $\left(4^{\prime \prime}\right) \Gamma(C \Rightarrow D, \neg D)$; then, we cut $\left(4^{\prime}\right)$ with (1) and $\left(4^{\prime \prime}\right)$ with (2) (inductive hypothesis for (a) on the height), obtaining:

$$
\text { (5) } \Gamma(C) \text { and }(6) \Gamma(\neg D) \text {. }
$$

Since $c p(C)=c^{\prime}<c p(C \Rightarrow D)=c$ we can use the inductive hypothesis on (c), namely $M P\left(c^{\prime}\right)$, so that from (5) and (3) we get

$$
\text { (7) } \Gamma(D)
$$

Since $c p(D)<c p(C \Rightarrow D)=c$ we can use the inductive hypothesis on (a), that is to say we cut $(6)$ and $(7)$ obtaining $\Gamma(\emptyset)$.

Concerning (b), the proof is the same as (B) of Theorem 16, except that there is an additional case where $\Gamma([A: \Delta])$ is derived by the $(M P)$ rule; we have two subcases: first $\Gamma([A: \Delta])=\Gamma^{\prime}(\neg(C \Rightarrow D),[A: \Delta])$ obtained by:

$$
\frac{\Gamma^{\prime}(\neg(C \Rightarrow D), C,[A: \Delta]) \quad \Gamma^{\prime}(\neg(C \Rightarrow D), \neg D,[A: \Delta])}{\Gamma^{\prime}(\neg(C \Rightarrow D),[A: \Delta])}(M P)
$$

We can obviously apply the inductive hypothesis to the two premises obtaining $\Gamma^{\prime}\left(\neg(C \Rightarrow D), C,\left[A^{\prime}: \Delta\right]\right)$ and $\Gamma^{\prime}\left(\neg(C \Rightarrow D), \neg D,\left[A^{\prime}: \Delta\right]\right)$ and then we conclude by an application of $(M P)$. The second subcase is when $\Gamma([A: \Delta])=\Gamma([A$ : $\left.\left.\neg(C \Rightarrow D), \Delta^{\prime}\right]\right)$ and is obtained by $(M P)$ as follows:

$$
\frac{\Gamma\left(\left[A: \neg(C \Rightarrow D), C, \Delta^{\prime}\right]\right) \quad \Gamma\left(\left[A: \neg(C \Rightarrow D), \neg D, \Delta^{\prime}\right]\right)}{\Gamma\left(\left[A: \neg(C \Rightarrow D), \Delta^{\prime}\right]\right)}(M P)
$$

and we proceed in the same way.

Concerning (c), it is proved by induction on the height $h$ of the derivation of $\Gamma([A: \Delta])$. For the base case $h=0$, we immediately conclude that, if $\Gamma([A: \Delta])$ is an axiom, so is $\Gamma(\Delta)$. For the inductive step, we distinguish the following subcases:

- $h>0$ : all cases, except $\left(\Rightarrow^{-}\right)$and $(I D)$, are straightforward, for instance if $\Gamma([A: \Delta])$ is obtained by:

$$
\frac{\Gamma_{1}([A: \Delta]) \quad \Gamma_{2}([A: \Delta])}{\Gamma([A: \Delta])}
$$

where $(\mathbf{R})$ is any rule different from $\left(\Rightarrow^{-}\right)$and $(I D)$, then we just apply the induction hypothesis to the premises obtaining $\Gamma_{1}(\Delta)$ and $\Gamma_{2}(\Delta)$ and then we apply $(\mathbf{R})$, we proceed similarly if $\Gamma([A: \Delta])$ is derived from say $\Gamma\left(\left[A: \Delta_{1}\right]\right)$ and $\Gamma\left(\left[A: \Delta_{2}\right]\right)$.

- $h>0\left(\Rightarrow^{-}\right)$, if $[A: \Delta]$ is not principal in the application of $\left(\Rightarrow^{-}\right)$, we proceed as above; the non-trivial case is when $\Gamma([A: \Delta])=\Gamma^{\prime}(\neg(C \Rightarrow D),[A: \Delta])$ and it is obtained as follows:

$$
\frac{(1) \Gamma^{\prime}(\neg(C \Rightarrow D),[A: \Delta, \neg D]) \quad(2) \neg A, C \quad \neg C, A}{\Gamma^{\prime}(\neg(C \Rightarrow D),[A: \Delta])}\left(\Rightarrow^{-}\right)
$$


In this case, by the property generalizing weakening of Lemma 10, from (2) we obtain a derivation of:

$$
\text { (3) } \Gamma^{\prime}(\neg(C \Rightarrow D), \neg A, C)
$$

We have that $\Gamma(A)$ is derivable (second condition in statement (c)), that is to say

$$
(*) \Gamma^{\prime}(\neg(C \Rightarrow D), A)
$$

is derivable. By Lemma 9 (weakening), also

$$
\text { (4) } \Gamma^{\prime}(\neg(C \Rightarrow D), A, C)
$$

is derivable. By inductive hypothesis on the complexity of the cut formula (i.e. the antecedent of statement (c)) we can cut (4) and (3) to obtain:

$$
\left(4^{\prime}\right) \Gamma^{\prime}(\neg(C \Rightarrow D), C),
$$

and by weakening

$$
\left(4^{\prime \prime}\right) \Gamma^{\prime}(\neg(C \Rightarrow D), \Delta, C)
$$

Now we apply the inductive hypothesis of (c) to (1) using the premise $(*) \Gamma^{\prime}(\neg(C \Rightarrow$ $D), A$ ) and we get

$$
\text { (5) } \Gamma(\neg(C \Rightarrow D), \Delta, \neg D)
$$

Now we apply the $(M P)$ rule to $\left(4^{\prime \prime}\right)$ and $(5)$ and we finally get:

$$
\Gamma^{\prime}(\neg(C \Rightarrow D), \Delta)
$$

- $h>0(I D)$, again the case is trivial if the inference step does not involve $[A: \Delta]$, the significant case is the following:

$$
\frac{(1) \Gamma([A: \Delta, \neg A])}{\Gamma([A: \Delta])}(I D)
$$

We apply the inductive hypothesis of (c) to (1) with the premise $\Gamma(A)$ and we get:

$$
\text { (2) } \Gamma(\Delta, \neg A)
$$

From $\Gamma(A)$, by weakening we have a derivation of $(3) \Gamma(\Delta, A)$, and we apply the inductive hypothesis on the complexity of the cut formula (i.e. the antecedent of statement (c)) to cut (2) and (3) and we finally get $\Gamma(\Delta)$.

\subsubsection{Completeness of $\mathcal{N} S$}

We can now prove the completeness of the calculi $\mathcal{N} S$ :

Theorem 18 (Completeness of $\mathcal{N} S$ ) If $\Gamma$ is valid, then it is derivable in $\mathcal{N} S$. 
Proof. We prove that the axioms are derivable and that the set of derivable formulas is closed under (Modus Ponens), (RCEA), and (RCK). A derivation of an instance of ID has been shown in Figure 2. A derivation of an instance of MP is as follows:

$$
\begin{aligned}
& \begin{array}{c}
\overline{\neg(A \Rightarrow B), \neg A, B, A}(A X) \overline{\neg(A \Rightarrow B), \neg A, B, \neg B} \\
(A X) \\
\neg(A \Rightarrow B), \neg A, B
\end{array} \\
& \frac{\frac{\neg(A \Rightarrow B), \neg A, B}{\neg(A \Rightarrow B), A \rightarrow B}\left(\rightarrow^{+}\right)}{(A \Rightarrow B) \rightarrow(A \rightarrow B)}\left(\rightarrow^{+}\right)
\end{aligned}
$$

Here is a derivation of an instance of CEM:

$$
\begin{gathered}
\frac{\overline{[A: B, \neg B]}(A X) \overline{A, \neg A}(A X) \overline{\neg A, A}(A X)}{\frac{[A: B],[A: \neg B]}{[A: B], A \Rightarrow \neg B}\left(\Rightarrow^{+}\right)}(C E M) \\
\frac{A \Rightarrow B, A \Rightarrow \neg B}{(A \Rightarrow B) \vee(A \Rightarrow \neg B)}\left(\Rightarrow^{+}\right) \\
\left(\mathrm{V}^{+}\right)
\end{gathered}
$$

For (Modus Ponens), we have to show that, if (1) $A \rightarrow B$ ad (2) $A$ are derivable, then also $B$ is derivable. Since weakening is admissible (Lemma 9), we have also derivations for $\left(1^{\prime}\right) A \rightarrow B, B, \neg A$ and $\left(2^{\prime}\right) A, B$. Furthermore, observe that (3) $A, B, \neg A$ and (4) $\neg B, B, \neg A$ are both instances of $(A X)$. Since cut is admissible (statement $\mathrm{A}$ in Theorem 16), the following derivation shows that $B$ is derivable:

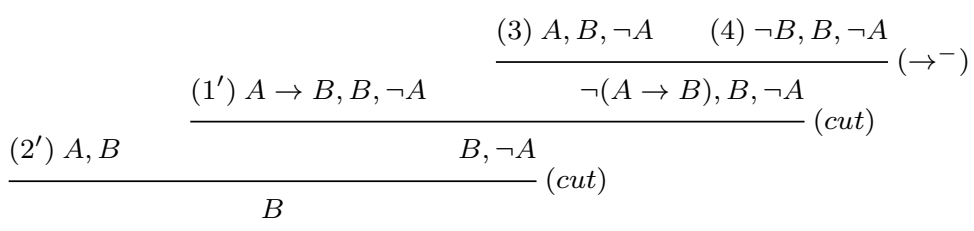

For (RCEA), we have to show that if $A \leftrightarrow B$ is derivable, then also $(A \Rightarrow C) \leftrightarrow$ $(B \Rightarrow C)$ is derivable. As usual, $A \leftrightarrow B$ is an abbreviation for $(A \rightarrow B) \wedge(B \rightarrow A)$. Since $A \leftrightarrow B$ is derivable, and since $\left(\wedge^{+}\right)$and $\left(\rightarrow^{+}\right)$are invertible (Lemma 11), we have a derivation for $A \rightarrow B$, then for (1) $\neg A, B$, and for $B \rightarrow A$, then for (2) $A, \neg B$. We derive $(A \Rightarrow C) \rightarrow(B \Rightarrow C)$ (the other half is symmetric) as follows:

$$
\frac{\overline{\neg(A \Rightarrow C),[B: C, \neg C]}(A X) \quad(1) \neg A, B \quad(2) A, \neg B}{\frac{\neg(A \Rightarrow C),[B: C]}{\frac{\neg(A \Rightarrow C), B \Rightarrow C}{(A \Rightarrow C) \rightarrow(B \Rightarrow C)}\left(\rightarrow^{+}\right)}\left(\rightarrow^{+}\right)}
$$

For $(\mathrm{RCK})$, suppose that we have a derivation in $\mathcal{N} S$ of $\left(A_{1} \wedge \ldots \wedge A_{n}\right) \rightarrow B$. Since $\left(\rightarrow^{+}\right)$is invertible (Lemma 11), we have also a derivation of $B, \neg\left(A_{1} \wedge \ldots \wedge A_{n}\right)$ Since $\left(\wedge^{-}\right)$is also invertible, then we have a derivation of $B, \neg A_{1}, \ldots, \neg A_{n}$ and, by Lemma 10 generalizing weakening, of $(1) \neg\left(C \Rightarrow A_{1}\right), \ldots, \neg\left(C \Rightarrow A_{n}\right),[C$ : 
$\left.B, \neg A_{1}, \neg A_{2}, \ldots, \neg A_{n}\right]$, from which we conclude as follows:

(1) $\neg\left(C \Rightarrow A_{1}\right), \ldots, \neg\left(C \Rightarrow A_{n}\right),\left[C: B, \neg A_{1}, \neg A_{2}, \ldots, \neg A_{n}\right]$

\begin{tabular}{|c|c|c|c|c|c|}
\hline \multicolumn{2}{|l|}{$\neg\left(C \Rightarrow A_{1}\right), \ldots, \neg\left(C \Rightarrow A_{n}\right),\left[C: B, \neg A_{1}, \neg A_{2}\right]$} & $\overline{C, \neg C}$ & $\overline{\neg C, C}$ & \multirow{2}{*}{$\overline{C, \neg C}(A X)$} & \multirow{2}{*}{$\overline{\neg C, C}(A X)$} \\
\hline \multicolumn{4}{|c|}{$\neg\left(C \Rightarrow A_{1}\right), \ldots, \neg\left(C \Rightarrow A_{n}\right),\left[C: B, \neg A_{1}\right]$} & & \\
\hline & \multicolumn{5}{|c|}{$\neg\left(C \Rightarrow A_{1}\right), \ldots, \neg\left(C \Rightarrow A_{n}\right),[C: B]$} \\
\hline & \multicolumn{5}{|c|}{  } \\
\hline & \multicolumn{5}{|c|}{$\neg\left(C \Rightarrow A_{1} \wedge \ldots \wedge C \Rightarrow A_{n}\right), C \Rightarrow B$} \\
\hline & $\left(C \Rightarrow A_{1} \wedge \ldots \wedge\right.$ & $\left(C \Rightarrow A_{n}\right) \rightarrow$ & $(C \Rightarrow B)$ & & \\
\hline
\end{tabular}

\subsection{Termination and complexity of $\mathcal{N} S$}

The rules $\left(\Rightarrow^{-}\right),(M P),(C E M)$, and (ID) may be applied infinitely often in a backward proof search, since their principal formula is copied into the premise(s). In order to obtain a terminating calculus, we have to put some restrictions on the application of these rules. We first consider the case of the calculi $\mathcal{N} S$ for systems without CEM, for which standard restrictions on the application of rules allow us to turn the calculi into terminating ones. For the systems allowing CEM, we need a more sophisticated machinery that we will describe in a separate section.

\subsubsection{Termination and complexity of $\mathcal{N} \mathrm{CK}, \mathcal{N} \mathrm{CK}+\mathrm{ID}, \mathcal{N} \mathrm{CK}+\mathrm{MP}$, and $\mathcal{N} \mathrm{CK}+\mathrm{ID}+\mathrm{MP}$}

We put the following restrictions:

Restrictions for terminating $\mathcal{N} C K, \mathcal{N} C K+I D, \mathcal{N} C K+M P$, and $\mathcal{N} C K+I D+M P$.

- apply $\left(\Rightarrow^{-}\right)$to $\Gamma\left(\neg(A \Rightarrow B),\left[A^{\prime}: \Delta\right]\right)$ only if $\left(\Rightarrow^{-}\right)$has not been applied to the formula $\neg(A \Rightarrow B)$ with the context $\left[A^{\prime}: \Delta\right]$ in the current branch;

- apply $(I D)$ to $\Gamma([A: \Delta])$ only if $(I D)$ has not been applied to the context $[A: \Delta]$ in the current branch;

- apply $(M P)$ to $\Gamma(\neg(A \Rightarrow B))$ only if $(M P)$ has not been applied to the formula $\neg(A \Rightarrow B)$ in the current branch.

These restrictions impose that $\left(\Rightarrow^{-}\right)$can be applied only once to each formula $\neg(A \Rightarrow$ $B)$ with a context $\left[A^{\prime}: \Delta\right]$ in each branch, that $(I D)$ can be applied only once to each context $[A: \Delta]$ in each branch, and that $(M P)$ can be applied only once to each formula $\neg(A \Rightarrow B)$ in each branch.

In order to obtain a terminating version of $\mathcal{N} S$, we need to implement the above restrictions. These restrictions can be effectively implemented by adding a suitable bookkeeping mechanism. The easiest way to do it (not necessarily the most efficient) is to equip sequents with three lists, let us call them COND, ID and MP, containing 
formulas and/or contexts already used in the current branch for an application of the three mentioned rules. For instance, the list CoND contains pairs $\left\langle\neg(A \Rightarrow B),\left[A^{\prime}\right.\right.$ : $\Delta]\rangle$ to keep track that, in the current branch, the rule $\left(\Rightarrow^{-}\right)$has been already applied to $\neg(A \Rightarrow B)$ by using $\left[A^{\prime}: \Delta\right]$, thus introducing the sequent $\Gamma\left(\neg(A \Rightarrow B),\left[A^{\prime}\right.\right.$ : $\Delta, \neg B])$. The rule $\left(\Rightarrow^{-}\right)$can be then applied to a sequent $\Gamma\left(\neg(A \Rightarrow B),\left[A^{\prime}: \Delta\right]\right)$ only if the pair $\left\langle\neg(A \Rightarrow B),\left[A^{\prime}: \Delta\right]\right\rangle$ does not belong to ConD. When the rule $\left(\Rightarrow^{-}\right)$ is applied to some $\Gamma\left(\neg(A \Rightarrow B),\left[A^{\prime}: \Delta\right]\right)$, then the pair $\left\langle\neg(A \Rightarrow B),\left[A^{\prime}: \Delta\right]\right\rangle$ is added to the list COND. The lists ID and MP, containing contexts $[A: \Delta]$ and negated conditional $\neg(A \Rightarrow B)$, respectively are used in a similar way to implement the restriction for $(I D)$ and $(M P)$.

Theorem 19 The calculi $\mathcal{N} S$ with the termination restrictions are sound and complete for their respective logics.

Proof. We show that it is useless to apply the rules $\left(\Rightarrow^{-}\right),(I D)$ and $(M P)$ without the restrictions.

- $\left(\Rightarrow^{-}\right)$: suppose it is applied twice on $\Gamma\left(\neg(A \Rightarrow B),\left[A^{\prime}: \Delta\right]\right)$ in a branch. Since $\left(\Rightarrow^{-}\right)$is "weakly" invertible (Lemma 12), we can assume, without loss of generality, that the two applications of $\left(\Rightarrow^{-}\right)$are consecutive, starting from $\Gamma\left(\neg(A \Rightarrow B),\left[A^{\prime}: \Delta, \neg B, \neg B\right]\right)$. By Lemma 13 (contraction), we have a derivation of $\Gamma\left(\neg(A \Rightarrow B),\left[A^{\prime}: \Delta, \neg B\right]\right)$, and we can conclude with a (single) application of $\left(\Rightarrow^{-}\right)$. Remember that contraction is rule-preserving admissible, therefore the obtained derivation does not add any application of $\left(\Rightarrow^{-}\right)$;

- (ID): similarly to the case of $\left(\Rightarrow^{-}\right)$above, suppose that the rule $(I D)$ is applied twice on $\Gamma([A: \Delta])$ in a branch. Since $(I D)$ is invertible (Lemma 11), we can assume, without loss of generality, that the two applications of $(I D)$ are consecutive, starting from $\Gamma([A: \Delta, \neg A, \neg A])$. As we have done for $\left(\Rightarrow^{-}\right)$, we conclude that the second application is useless, since we obtain a derivation of $\Gamma([A: \Delta, \neg A])$ since contraction is admissible (remember again that contraction is rule-preserving admissible), and we get $\Gamma([A: \Delta])$ with a single application of $(I D)$;

- $(M P)$ : similarly to the cases above, suppose that the rule $(M P)$ is applied twice on $\Gamma(\neg(A \Rightarrow B))$ in a branch. Since $(M P)$ is invertible (Lemma 11), we can assume, without loss of generality, that the two applications of $(M P)$ are consecutive, starting from $\Gamma(\neg(A \Rightarrow B), A, A)$ and from $\Gamma(\neg(A \Rightarrow B), \neg B, \neg B)$. As we have done for $\left(\Rightarrow^{-}\right)$and $(I D)$, we conclude that the second application is useless: we obtain derivations of $\Gamma(\neg(A \Rightarrow B), A)$ and $\Gamma(\neg(A \Rightarrow B), \neg B)$ since contraction is rule-preserving admissible, then we get $\Gamma(\neg(A \Rightarrow B))$ with a single application of $(M P)$.

The above restrictions ensure a terminating proof search for the systems under consideration, in particular:

Theorem 20 The calculi $\mathcal{N} C K, \mathcal{N} C K+I D, \mathcal{N} C K+M P$, and $\mathcal{N} C K+I D+M P$ with the termination restrictions give a PSPACE decision procedure for their respective logics. 
To prove Theorem 20, we first show that the size of any sequent occurring in a derivation is polynomially bounded by the size of the initial sequent. We then give a polynomial bound on the number of rule applications in any branch of the derivation. By these results, we obtain that the size of every branch in a derivation is polynomially bounded by the size of the sequent at the root. As a consequence, the calculi $\mathcal{N} S$ with the termination restriction provides a PSPACE decision procedure.

From now on, we denote by $\operatorname{sub}(\Gamma)$ the set of subformulas of $\Gamma$. First of all, we need to define the conditional depth of a formula $F \in \operatorname{sub}(\Gamma)$. Intuitively, we say that $F$ has conditional depth $n$ (or simply has depth $n$ ) if $F$ or its negation appears in $\Gamma$ with a conditional nesting of $n$. Before introducing the depth of a formula we further need to define the notion of propositional subformula:

Definition 21 (Propositional subformula) Given a formula $F \in \mathcal{L}$, we define the notion of propositional subformula $(P S)$ of $F$ as follows:

(i) F is a PS of F;

(ii) $F$ is a $P S$ of $\neg F$;

(iii) if $F$ is a PS of $A$, then $F$ is a PS of $A \otimes B$, where $\otimes \in\{\wedge, \vee, \rightarrow\}$;

(iv) if $F$ is a PS of $B$, then $F$ is a PS of $A \otimes B$, where $\otimes \in\{\wedge, \vee, \rightarrow\}$.

Let $\Gamma$ be a sequent and let $F$ be a formula in $\operatorname{sub}(\Gamma)$. We say that $F$ is a propositional subformula of $\Gamma$ if there is $G \in \Gamma$ such that $F$ is a propositional subformula of $G$.

Definition 22 (Conditional depth of a formula (depth of a formula)) Let $\Gamma$ be a sequent. Let $F$ be a formula in $\mathrm{sub}(\Gamma)$. We define the conditional depth (or simply depth) of $F$ as follows:

- if $F$ is a propositional subformula of $\Gamma$ (Definition 21), then $F$ has conditional depth 0 ;

- given $A \Rightarrow B$ (resp. $\neg(A \Rightarrow B)$ ) a propositional subformula of $\Gamma$ with conditional depth $n$, if $F$ is a propositional subformula of either $A$ or $B$, then $F$ has conditional depth $n+1$.

Note that a formula can have multiple depths: for instance if $\Gamma=B \vee((A \vee C) \Rightarrow$ $(B \vee A \vee(C \Rightarrow B)))$, then $B$ has depths 0,1 and 2, whereas $A$ has depth 1 and $C$ has depths 1 and 2 . Let $\operatorname{depth}(\Gamma, n)$ be the set of formulas of $\operatorname{sub}(\Gamma)$ of depth $n$. We have $\Sigma_{i=0}^{\infty}|\operatorname{depth}(\Gamma, i)|=\mathrm{O}(|\Gamma|)$.

Now to give bounds to the size of a sequent, and to the size of a derivation, we have to give a bound to the number of contexts (possibly nested) that can appear in a sequent $\Lambda$ occurring in $\Pi$.

By induction on the level of nesting $n$ of a context $[A: \Delta]$, we can show that $\Delta$ can only contain formulas whose conditional depth in $\Gamma$ is $n+1$. Indeed, observe that a formula $B \in \Delta$ can only come from i) an application of a boolean rule which does not change the depth of a formula ii) an application of $\left(\Rightarrow^{+}\right)$, hence it comes from a formula $A \Rightarrow B$ with a nesting level of $n-1$, iii) an application of $\left(\Rightarrow^{-}\right)$ or $(M P)$, hence it comes from a formula $\neg\left(A^{\prime} \Rightarrow C\right)$, with a nesting level of $n-1$, where $B \equiv \neg C$, iv) $(I D)$, here $A=\neg B$ and since the context has been necessarily introduced by an application of $\left(\Rightarrow^{+}\right)$to a formula $(A \Rightarrow C)$ of depth $n-1$, we easily 
conclude that $\neg B$ has depth $n$. Therefore, since contexts are only created by the rule $\left(\Rightarrow^{+}\right)$, the number of immediate sub-contexts of a context $[A: \Delta]$ at a depth $n$ is bounded by the number of positive conditional formulas of depth $n+1$ occurring in $\operatorname{sub}(\Gamma)$. By an easy induction, we can show that the maximum number of contexts that can appear in a sequent $\Lambda \in \Pi$ is bounded by $\sum_{i=0}^{\infty}|\operatorname{depth}(\Gamma, i)| \times|\operatorname{depth}(\Gamma, i+1)|$, which is in $\mathrm{O}\left(|\Gamma|^{2}\right)$.

Lemma 23 Let $\Pi$ be a derivation of a sequent $\Gamma$ in $\mathcal{N} S$ with the termination restrictions, and $\Lambda$ be a sequent occurring in $\Pi$. Then $|\Lambda| \in \mathrm{O}\left(|\Gamma|^{3}\right)$.

Proof. We first give a bound on the size of each context. Let $[A: \Delta]$ be a context with a nesting level of $n$. By our previous discussion, $\Delta$ can only contain formulas of depth $n$. The termination restrictions preventing redundant application of the rules, $|\Delta|$ is linearly bounded by $|\operatorname{depth}(\Gamma, n)|$, which is itself bounded by $|\Gamma|$.

We also have to take into account the extra space needed to implement the termination restrictions. These restrictions can be implemented by some bookkeeping mechanism; instead of using global lists as described at the beginning of this section, we can proceed more economically as follows: for each context we store the list of (negative conditional) formulas to which the rule $\left(\Rightarrow^{-}\right)$has been applied with this context. Since this rule can only be applied once to each formula with respect to a given context, the extra space overhead is in $\mathrm{O}(|\Gamma|)$. For the rules $(I D)$ and $(M P)$, we can simply use a flag to record whether the rules have been applied to a given context/negated conditional. This gives a constant space overhead for each context. Therefore, the space needed to store a single context (taking the termination restrictions into account) is in $\mathrm{O}(|\Gamma|)$. The number of (possibly nested) contexts that can appear in $\Lambda$ being in $\mathrm{O}\left(|\Gamma|^{2}\right)$, we conclude that $|\Lambda| \in \mathrm{O}\left(|\Gamma|^{3}\right)$.

We now bound the length of a branch in a derivation.

Lemma 24 Let $\Pi$ be a derivation of a sequent $\Gamma$ in $\mathcal{N} S$ with the termination restrictions. Then the number of rule applications in any given branch of $\Pi$ is in $\mathrm{O}\left(|\Gamma|^{4}\right)$.

Proof. Let $[A: \Delta]$ be a context occurring in $\Lambda$ with a nesting level of $n$. We estimate the number of rules that can be applied within this context, i.e. the number of rules that can be applied to formulas of $\Delta$. We observe that: 1. As usual, the boolean rules cannot be applied redundantly and the number of applications of these rules is linearly bounded by the number of boolean subformulas occurring in $\Delta$, which is itself linearly bounded by $|\Gamma|$. 2. The rules $\left(\Rightarrow^{+}\right)$can be applied at most once for each positive conditional formula of depth $n$ appearing in subformulas of $\Delta$. Hence its number of applications is linearly bounded by $|\Gamma|$. Since this is the only rule which can introduce new contexts, this also gives a (linear) bound to the number of contexts that can appear in $\Delta$. 3. Due to the termination restrictions, the rule $\left(\Rightarrow^{-}\right)$can be applied once for each negative conditional formula of depth $n$ appearing in subformulas of $\Delta$, and for each context occurring in $\Delta$. Both numbers being linearly bounded by $|\Gamma|$, the number of applications of the rule $\left(\Rightarrow^{-}\right)$is linearly bounded by $|\Gamma|^{2}$. 4. The rule $(I D)$ can be applied at most once for each context in $\Delta$ (due to termination restrictions), and thus its number of applications is linearly bounded by $|\Gamma|$. 5. The rule $(M P)$ can be applied at most once for each negative conditional formula of depth $n$ appearing in subformulas 
of $\Delta$ (due to termination restrictions), and thus its number of applications is linearly bounded by $|\Gamma|$.

Therefore the number of rule applications in a given context is linearly bounded by $|\Gamma|^{2}$. The number of (possibly nested) contexts that can appear in $\Lambda$ being bounded by $|\Gamma|^{2}$, we obtain the linear bound of $|\Gamma|^{4}$ to the number of applications of rules in any branch of the derivation.

By the bounds on the size of a sequent and the number of rule applications in a given branch of a derivation, we obtain that the size of each branch is in $\mathrm{O}\left(|\Gamma|^{7}\right)$ and Theorem 20 follows.

\subsubsection{Termination and complexity of $\mathcal{N} \mathrm{CK}+\mathrm{CEM}$ and $\mathcal{N} \mathrm{CK}+\mathrm{CEM}+\mathrm{ID}$}

In order to prove that we can turn the calculus into a terminating one, we need the following definitions.

Definition 25 Given a nested sequent $\Gamma=A_{1}, \ldots, A_{n},\left[B_{1}: \Gamma_{1}\right], \ldots,\left[B_{m}: \Gamma_{m}\right]$ and a formula $F$, we define

$$
F \in \in^{*} \Gamma
$$

if either $F=A_{i}$ for some $i \in\{1,2, \ldots, n\}$ or if $F \in^{*} \Gamma_{j}$ for some $j \in\{1,2, \ldots, m\}$.

Definition 26 (CEM-reduced sequent) Given a nested sequent $\Gamma=A_{1}, \ldots, A_{n},\left[B_{1}\right.$ : $\left.\Gamma_{1}\right], \ldots,\left[B_{m}: \Gamma_{m}\right]$ occurring in $\Pi$, we say that $\Gamma$ is $\mathrm{CEM}$-reduced if the following conditions hold:

- for each formula $F$ such that $F \in^{*} \Gamma$, either $F$ is a literal, i.e. $F=P$ or $F=\neg P$, where $P \in A T M$, or $F$ is a negated conditional $\neg(C \Rightarrow D)$;

- for each negated conditional $\neg(C \Rightarrow D) \in^{*} \Gamma$ and for each (sub)context $\left[C^{\prime}: \Delta\right]$ occurring in $\Gamma$, if $C, \neg C^{\prime}$ and $C^{\prime}, \neg C$ are derivable, then the rule $\left(\Rightarrow^{-}\right)$ has been applied to $\neg(C \Rightarrow D)$ by using $\left[C^{\prime}: \Delta\right]$ in $\Pi$.

The intuitive idea is that, given a CEM-reduced sequent, we have already applied $\left(\Rightarrow^{-}\right)$ as much as possible to each negated conditional $\neg(C \Rightarrow D)$. Furthermore, since it contains only atomic formulas and negated conditionals, all subformulas of $\neg D$ have been introduced in the involved contexts.

Let us now introduce a nested inclusion among nested sequents:

Definition 27 Given two sequents $\Gamma=A_{1}, \ldots, A_{n},\left[B_{1}: \Gamma_{1}\right], \ldots,\left[B_{m}: \Gamma_{m}\right]$ and $\Delta$, we define

$$
\Gamma \subseteq \subseteq^{*} \Delta
$$

if the following conditions hold:

- $A_{i} \in \Delta$, for each $i=1,2, \ldots, n$

- there is $\left[B_{i}: \Delta_{i}\right] \in \Delta$ such that $\Gamma_{i} \subseteq^{*} \Delta_{i}$, for each $i=1,2, \ldots, m$.

As an example, the following sequents: 


$$
\begin{aligned}
& \Gamma=C, C,[D: E, E, F],[D: E, F],[G:[H: K, K]] \\
& \Delta=C,[D: E, E, F, H],[G:[H: K, M]]
\end{aligned}
$$

are such that $\Gamma \subseteq \subseteq^{*} \Delta$.

In order to obtain a terminating procedure, we have to put the following restrictions on the application of some rules:

\section{Restrictions for terminating $\mathcal{N} C K+C E M$ and $\mathcal{N} C K+C E M+I D$.}

- apply $\left(\Rightarrow^{-}\right)$to $\Gamma\left(\neg(A \Rightarrow B),\left[A^{\prime}: \Delta\right]\right)$ only if $\left(\Rightarrow^{-}\right)$has not been applied to the formula $\neg(A \Rightarrow B)$ with the context $\left[A^{\prime}: \Delta\right]$ in the current branch;

- apply $(I D)$ to $\Gamma([A: \Delta])$ only if $(I D)$ has not been applied to $[A: \Delta]$ in the current branch;

- apply $(C E M)$ to $\Gamma([A: \Delta],[B: \Sigma])$ only if

1. $\Gamma([A: \Delta],[B: \Sigma])$ is CEM-reduced

2. $\operatorname{not} \Sigma \subseteq \subseteq^{*} \Delta$.

These restrictions impose that $\left(\Rightarrow^{-}\right)$is applied only once to a given formula $\neg(A \Rightarrow B)$ with a context $\left[A^{\prime}: \Delta\right]$ in each branch and that $(I D)$ is applied only once to a given context $[A: \Delta]$. Furthermore, also $(C E M)$ is applied a finite number of times in a given branch. This is stated in a rigorous manner in the following theorems.

Theorem 28 The calculi $\mathcal{N} C K+C E M$ and $\mathcal{N} C K+C E M+I D$ with the termination restrictions is sound and complete for their respective logics.

Proof. We show that it is useless to apply the rules $\left(\Rightarrow^{-}\right),(I D)$ and $(C E M)$ without the restrictions. We proceed by induction on the height of the derivation of a valid sequent $\Gamma$. For the cases of $\left(\Rightarrow^{-}\right)$and $(I D)$ we reason as in the proof of Theorem 6 .

Let us now consider the non-trivial case of $(C E M)$. We distinguish two cases, namely an application of $(C E M)$ violating the termination condition number 1 and an application of $(C E M)$ violating the termination condition number 2.

1. let us consider a derivation containing an application of $(C E M)$ to a non CEMreduced sequent, as follows:

$$
\frac{\Gamma([A: \Delta, \Sigma],[B: \Sigma]) \quad A, \neg B \quad B, \neg A}{\Gamma([A: \Delta],[B: \Sigma])}(C E M)
$$

Since the rules are invertible (Lemma 11) or, at least, "weakly" invertible ${ }^{2}$, we can permute the application of the rules in $\Pi$ over $(C E M)$. As an example, consider a derivation $\Pi$ ending with an application of $\left(\Rightarrow^{-}\right)$as follows:

\footnotetext{
${ }^{2}$ The rules $\left(\Rightarrow^{-}\right)$and $(C E M)$ are not invertible, however, their leftmost premises can be obtained by weakening from the respective conclusions.
} 


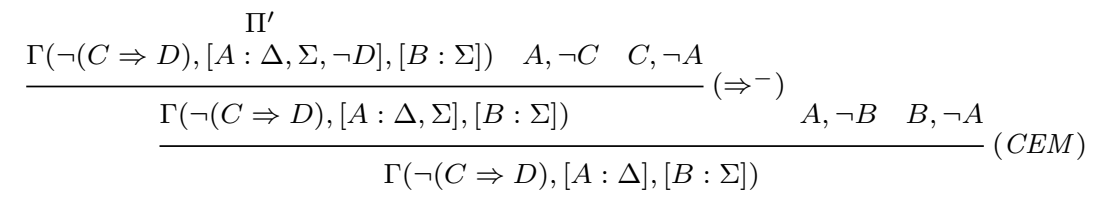

We obtain the following derivation:

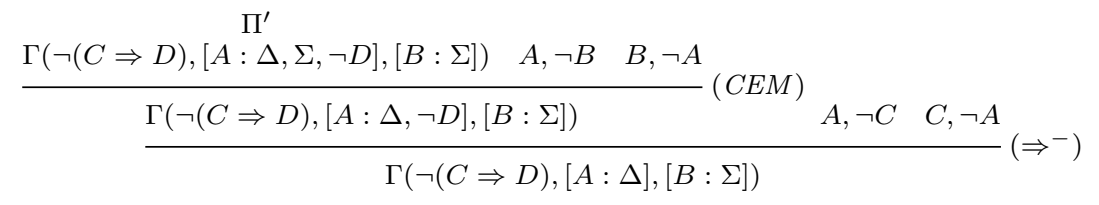

We can therefore permute all the rules, obtaining a derivation in which $(C E M)$ is applied to CEM-reduced sequents only.

2. let us consider a derivation containing an application of $(C E M)$ to $\Gamma([A$ : $\Delta],[B: \Sigma])$ such that $\Sigma \subseteq^{*} \Delta$. We have a derivation as follows:

$$
\frac{(1) \Gamma([A: \Delta, \Sigma],[B: \Sigma]) \quad A, \neg B \quad B, \neg A}{\left(1^{\prime}\right) \Gamma([A: \Delta],[B: \Sigma])}(C E M)
$$

Since $\Sigma \subseteq^{*} \Delta$, from (1) and the fact that contraction is height-preserving admissible (Lemma 13), we immediately get that there is a derivation whose height is no greater than (1)'s, also of $\left(1^{\prime}\right)$, therefore the application of $(C E M)$ is useless.

One might have the suspect that the above restrictions on the application of $(C E M)$ are not sufficient to ensure termination due to the fact that such a rule can be applied to $\Gamma([A: \Delta],[B: \Sigma])$ in two different ways, namely:

$$
\begin{aligned}
& \frac{\Gamma([A: \Delta, \Sigma],[B: \Sigma]) \quad A, \neg B \quad B, \neg A}{\Gamma([A: \Delta],[B: \Sigma])}(C E M) \\
& \frac{\Gamma([A: \Delta],[B: \Delta, \Sigma]) \quad A, \neg B \quad B, \neg A}{\Gamma([A: \Delta],[B: \Sigma])}(C E M)
\end{aligned}
$$

We observe that this is not the case. Indeed, let us consider the former derivation: the restriction 2 does not avoid a further application of (CEM) (the "symmetric one"), as follows:

$$
\frac{(*) \Gamma([A: \Delta, \Sigma],[B: \Delta, \Sigma, \Sigma]) \quad A, \neg B \quad B, \neg A}{\frac{\Gamma([A: \Delta, \Sigma],[B: \Sigma])}{\Gamma(C E M)} A, \neg B \quad B, \neg A}(C E M)
$$


However, the rule $(C E M)$ is no longer applicable to $(*)$, because both $\Delta, \Sigma \subseteq^{*}$ $\Delta, \Sigma, \Sigma$ and $\Delta, \Sigma, \Sigma \subseteq^{*} \Delta, \Sigma$ hold.

Now we have all the ingredients to prove that the calculi $\mathcal{N C K}+\mathrm{CEM}+\mathrm{ID}$ and $\mathcal{N C K}+\mathrm{CEM}$ ensure a terminating proof search. Moreover, we are able to give a PSPACE complexity upper bound for the respective logics.

Theorem 29 The calculi $\mathcal{N} C K+C E M$ and $\mathcal{N} C K+C E M+I D$ with the termination restrictions give a PSPACE decision procedure for their respective logics.

Proceeding similarly to what done for systems not allowing CEM, we can prove that:

Lemma 30 Let $\Pi$ be a derivation of a sequent $\Gamma$ in $\mathcal{N} C K+C E M$ and $\mathcal{N} C K+C E M+I D$ with the termination restrictions, and $\Lambda$ be a sequent occurring in $\Pi$. Then $|\Lambda| \in$ $\mathrm{O}\left(|\Gamma|^{4}\right)$.

Proof. The proof is very similar to the one of Lemma 23 above. We first give a bound on the size of each context. Let $[A: \Delta]$ be a context with a nesting level of $n$. By our previous discussion, $\Delta$ can only contain formulas of depth $n$. The termination restrictions preventing redundant application of the rules, $|\Delta|$ is linearly bounded by $|\operatorname{depth}(\Gamma, n)|$, which is itself bounded by $|\Gamma|$.

We also have to take into account the extra space that may be needed to implement the termination restrictions. Observe that these restrictions can be implemented by some bookkeeping mechanism, that is for each context we store the list of (negative conditional) formulas to which the rule $\left(\Rightarrow^{-}\right)$has been applied with this context. Since this rule can only be applied once to each formula with respect to a given context, the extra space overhead is in $\mathrm{O}(|\Gamma|)$. Similarly for the rule $(C E M)$ : since this rule can only be applied once to each pair of contexts $[A: \Gamma]-[B: \Sigma]$, the extra space overhead is in $\mathrm{O}\left(|\Gamma|^{2}\right)$. For the rule $(I D)$, we can simply use a flag to record whether the rule has been applied to a given context. This gives a constant space overhead for each context. Therefore, the space needed to store a single context (taking the termination restrictions into account) is in $\mathrm{O}\left(|\Gamma|^{2}\right)$. The number of (possibly nested) contexts that can appear in $\Lambda$ being in $\mathrm{O}\left(|\Gamma|^{2}\right)$, we conclude that $|\Lambda| \in \mathrm{O}\left(|\Gamma|^{4}\right)$.

Lemma 31 Let $\Pi$ be a derivation of a sequent $\Gamma$ in $\mathcal{N} C K+C E M$ and $\mathcal{N} C K+C E M+I D$ with the termination restrictions. Then the number of rule applications in any given branch of $\Pi$ is in $\mathrm{O}\left(|\Gamma|^{6}\right)$.

Proof. The proof is the same as the one for Lemma 24. We just further observe that the rule $(C E M)$ "merges" contexts ${ }^{3}$ occurring in $\Delta$, and thus its number of applications is linearly bounded by the number of contexts that can appear in $\Delta$, that is it is linearly bounded by $|\Gamma|^{2}$. Therefore the number of rule applications in a given context is linearly bounded by $|\Gamma|^{4}$. The number of (possibly nested) contexts that can appear in $\Lambda$ being bounded by $|\Gamma|^{2}$, we easily obtain the linear bound of $|\Gamma|^{6}$ to the number of applications of rules in any branch of the derivation.

By the bounds on the size of a sequent and the number of rule applications in a given branch of a derivation, we obtain that the size of each branch is in $\mathrm{O}\left(|\Gamma|^{7}\right)$ and

\footnotetext{
${ }^{3}$ We mean that, given a backward application of $(C E M)$ to $\Gamma([A: \Delta],[B: \Sigma]), \Delta$ and $\Sigma$ are "merged" into the leftmost premise of the rule in the context with $A$, namely we have that $\Gamma([A: \Delta, \Sigma],[B: \Sigma])$.
} 
Theorem 29 follows. It is worth noticing that our calculi match the PSPACE lowerbound of the logics CK and CK+ID, and are thus optimal with respect to these logics. On the contrary the calculi for $\mathrm{CK}+\mathrm{CEM}(+\mathrm{ID})$ are not optimal, since validity in these logics is known to be decidable in CONP. In future work we shall try to devise an optimal decision procedure by adopting a suitable strategy.

\section{A calculus for the KLM Cumulative Logic C}

In this section we show another application of nested sequents to give an analytic calculus for the flat fragment, i.e. without nested conditionals $\Rightarrow$, of $\mathrm{CK}+\mathrm{CSO}+\mathrm{ID}$. This logic is well-known and it corresponds to logic $\mathbf{C}$, the logic of cumulativity, the weakest system in the family of KLM logics [22]. Formulas are restricted to boolean combinations of propositional formulas and conditionals $A \Rightarrow B$ where $A$ and $B$ are propositionals.

Kraus, Lehmann and Magidor have given the following axiomatization for cumulative logic $\mathbf{C}\left(\vdash_{P C}\right.$ denotes provability in propositional logic):

- any axiomatization of the classical propositional calculus

(prop)

- $A \Rightarrow A$

- If $\vdash_{P C} A \leftrightarrow B$, then $\vdash(A \Rightarrow C) \rightarrow(B \Rightarrow C)$

- If $\vdash_{P C} A \rightarrow B$, then $\vdash(C \Rightarrow A) \rightarrow(C \Rightarrow B)$

- $((A \Rightarrow B) \wedge(A \Rightarrow C)) \rightarrow(A \wedge B \Rightarrow C)$

- $((A \Rightarrow B) \wedge(A \Rightarrow C)) \rightarrow(A \Rightarrow B \wedge C)$

- $((A \Rightarrow B) \wedge(A \wedge B \Rightarrow C)) \rightarrow(A \Rightarrow C)$

The logic $\mathbf{C}$ has also an alternative semantics in terms of weak preferential models that is worthwhile to recall:

Definition 32 (Preferential Semantics of C, Definitions 5, 6, 7 in [22]) A cumulative model is a tuple

$$
\mathcal{M}=\langle S, \mathcal{W}, l,<, V\rangle
$$

where $S, \mathcal{W}, l$, and $V$ are defined as follows:

- $S$ is a set of elements are called states;

- $\mathcal{W}$ is a set of possible worlds;

- $l: S \longmapsto 2^{\mathcal{W}}$ is a function that labels every state with a nonempty set of worlds;

- $<$ is an irreflexive ${ }^{4}$ relation on $S$;

- $V$ is a valuation function $V: \mathcal{W} \longmapsto 2^{A T M}$, which assigns to every world $w$ the atoms holding in that world.

\footnotetext{
${ }^{4}$ In Definition 5 in [22] the relation $<$ is not explicitly assumed to be irreflexive. However, it is assumed that it satisfies the smoothness condition, and this implies that the relation is also irreflexive.
} 
For $s \in S$ and A propositional, let $\mathcal{M}, s \mid \equiv A$ if $\forall w \in l(s), \mathcal{M}, w \models A$, where $\mathcal{M}, w=A$ is defined as in propositional logic. Let $\operatorname{Min}_{<}(A)$ be the set of minimal states $s$ such that $\mathcal{M}, s \equiv A$. It is defined that $\mathcal{M}, s \mid \equiv A \sim B$ if $\forall s^{\prime} \in \operatorname{Min}_{<}(A)$, $\mathcal{M}, s^{\prime} \equiv B$. The relation $\equiv$ is extended to boolean combinations of conditionals in the standard way. The relation < satisfies the following smoothness condition: given a propositional formula $A$, a model $\mathcal{M}$ and a state $s \in S$, if $\mathcal{M}, s \mid \equiv A$, then either $s \in \operatorname{Min}_{<}(A)$ or there exists $s^{\prime} \in S$ such that $s^{\prime}<s$ and $s^{\prime} \in \operatorname{Min}_{<}(A)$.

Given a model $\mathcal{M}=\langle S, \mathcal{W}, l,<, V\rangle$, a formula $F$ is valid in $\mathcal{M}$ if $\mathcal{M}, s \equiv A$ for every $s \in S$ (written $\mathcal{M}=F$ ). A formula $F$ is valid if it is valid in every model, i.e. $\mathcal{M} \models F$ for every model $\mathcal{M}$ (written $\models F$ ).

In [22] it is shown that the above axiomatization is sound and complete with respect to the semantics of Definition 32:

Theorem 33 (Soundness and completeness of the axiomatization w.r.t. the semantics of Definition 32 [22]) Given a formula $F, F$ is valid in $\mathbf{C}$ if and only if it is provable in the axiomatization, i.e. $\models F$ if and only if $\vdash F$.

In [11] it has been shown that KLM logic $\mathbf{C}$ corresponds to the flat fragment of conditional logic $\mathrm{CK}+\mathrm{CSO}+\mathrm{ID}^{5}$, thus showing an alternative axiomatization of $\mathbf{C}$ given by the axioms and rules (prop), (Modus Ponens), (RCEA), (RCK), ID, and CSO introduced in Section 2 above.

We are able to extend our nested sequents calculi to deal also with the flat fragment of $\mathrm{CK}+\mathrm{ID}+\mathrm{CSO}$. A sequent has then the form:

$$
A_{1}, \ldots, A_{m},\left[B_{1}: \Delta_{1}\right], \ldots,\left[B_{m}: \Delta_{m}\right]
$$

where $B_{i}$ and $\Delta_{i}$ are propositional. The rules of $\mathcal{N} \mathbf{C}_{\mathrm{KLM}}$ are those ones of $\mathcal{N} \mathrm{CK}+\mathrm{ID}$ (restricted to the flat fragment) where the rule $\left(\Rightarrow^{-}\right)$is replaced by the rule $(C S O)$ :

$$
\frac{\Gamma, \neg(C \Rightarrow D),[A: \Delta, \neg D] \quad \Gamma, \neg(C \Rightarrow D),[A: C] \quad \Gamma, \neg(C \Rightarrow D),[C: A]}{\Gamma, \neg(C \Rightarrow D),[A: \Delta]}
$$

The calculi $\mathcal{N} \mathbf{C}_{\mathrm{KLM}}$ are shown in Figure 3. A derivation of an instance of CSO is shown in Figure 4. More interestingly, in Figure 5 we give an example of derivation of $\mathrm{CM}$ (cumulative monotony)

$$
\mathrm{CM} \quad((A \Rightarrow B) \wedge(A \Rightarrow C)) \rightarrow(A \wedge B \Rightarrow C) .
$$

As for the calculi $\mathcal{N} S$, we can prove that weakening is height preserving admissible also in the calculus $\mathcal{N} \mathbf{C}_{\mathrm{KLM}}$ for $\mathbf{C}$ :

\footnotetext{
${ }^{5}$ In [11] it is shown that KLM's logic C corresponds to the flat fragment of CK+ID+AC+RT which, in turn, corresponds to $\mathrm{CK}+\mathrm{ID}+\mathrm{CSO}$. This can be easily proved by showing the interderivability between the $\mathrm{CSO}$ axiom, on the one hand, and the $\mathrm{AC}+\mathrm{RT}$ axioms, on the other hand, or by showing the equivalence between the corresponding semantic conditions on the selection function: on the one hand, in models with $\mathrm{AC}$ and RT, if $f(w,[A]) \subseteq[B]$, then $f(w,[A])=f(w,[B])$. Indeed, if $f(w,[A]) \subseteq[B]$, by AC we have that $f(w,[A \wedge B]) \subseteq f(w,[A])$, and by RT we have that $(w,[A]) \subseteq f(w,[A \wedge B])$. Therefore in a $\mathrm{CK}+\mathrm{ID}+\mathrm{AC}+\mathrm{RT}$ model the semantic condition corresponding to CSO, namely if $f(w,[A]) \subseteq[B]$ and $f(w,[B]) \subseteq[A]$ then $f(w,[A])=f(w,[B])$, holds. On the other hand, in a CK+CSO+ID model, if $f(w,[A]) \subseteq[B]$, we have that $f(w,[A]) \subseteq[A]$ by ID, then $(*) f(w,[A]) \subseteq[A \wedge B]$. Again by ID, we have that $(* *) f(w,[A \wedge B]) \subseteq[A \wedge B] \subseteq[A]$. By CSO, from $(*)$ and $(* *)$ we conclude that $f(w,[A])=f(w,[A \wedge B])$, i.e. the conditions corresponding to both $\mathrm{AC}$ and $\mathrm{RT}$ hold.
} 


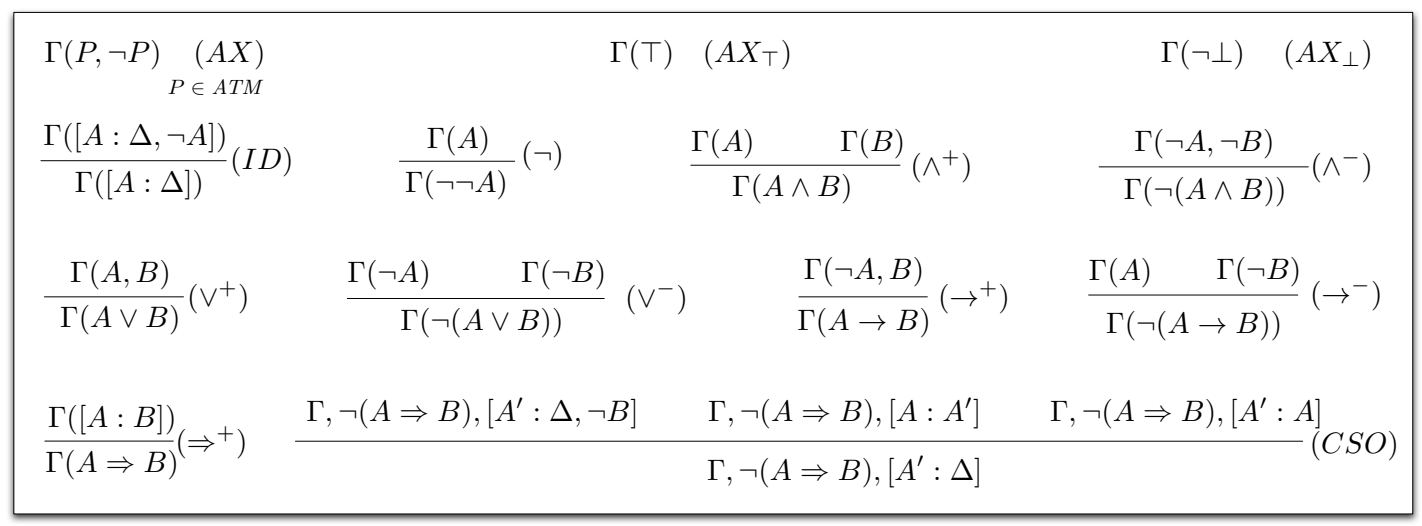

Figure 3: The nested sequent calculi $\mathcal{N} \mathbf{C}_{\mathrm{KLM}}$.

Lemma 34 (Admissibility of weakening in $\mathcal{N} \mathbf{C}_{\mathbf{K L M}}$ ) Weakening is height-preserving admissible in $\mathcal{N} \mathbf{C}_{K L M}$ : if $\Gamma(\Delta)$ is derivable in $\mathcal{N} S$ with a derivation of height $h$, then also $\Gamma(\Delta, \Sigma)$ is derivable in $\mathcal{N} S$ with a proof of height $h^{\prime} \leq h$, where $\Delta$ and $\Sigma$ are nested sequents.

Proof. Exactly as we have done for $\mathcal{N} S$, we proceed by induction on the height $h$ of the derivation of $\Gamma(\Delta)$. The only case we have to consider further is when the derivation is ended by an application of ( $C S O)$, as follows:

$$
\frac{(1) \Gamma, \neg(C \Rightarrow D),[A: \Delta, \neg D] \quad(2) \Gamma, \neg(C \Rightarrow D),[A: C] \quad(3) \Gamma, \neg(C \Rightarrow D),[C: A]}{\Gamma, \neg(C \Rightarrow D),[A: \Delta]}(C S O)
$$

We can apply the inductive hypothesis to the three premises, to obtain derivations of $\left(1^{\prime}\right) \Gamma, \Sigma, \neg(C \Rightarrow D),[A: \Delta, \neg D]$ (respectively, of $\left(1^{\prime \prime}\right) \Gamma, \neg(C \Rightarrow D),[A$ : $\Delta, \Sigma, \neg D]),\left(2^{\prime}\right) \Gamma, \Sigma, \neg(C \Rightarrow D),[A: C]$, and $\left(3^{\prime}\right) \Gamma, \Sigma, \neg(C \Rightarrow D),[C: A]$, from which we obtain a derivation of $\Gamma, \Sigma, \neg(C \Rightarrow D),[A: \Delta]$ by applying $(C S O)$ to $\left(1^{\prime}\right)$, $\left(2^{\prime}\right)$ and $\left(3^{\prime}\right)$, as well as of $\Gamma, \neg(C \Rightarrow D),[A: \Delta, \Sigma]$ by applying $(C S O)$ to $\left(1^{\prime \prime}\right),(2)$ and $(3)$.

Exactly as done for Lemma 10, we can prove that the generalization of wekening also holds in $\mathcal{N} \mathbf{C}_{\mathrm{KLM}}$ :

Lemma 35 Let $\Delta$ be a nested sequent and let $\Gamma()$ be a context. If $\Delta$ is derivable in $\mathcal{N} \mathbf{C}_{K L M}$, then also $\Gamma(\Delta)$ is derivable in $\mathcal{N} \mathbf{C}_{K L M}$.

Furthermore, it is easy to observe that the rule $(C S O)$ is "weakly" invertible, since its leftmost premise is obtained by weakening from the conclusion.

Our goal is to prove that contraction is admissible also in the calculus $\mathcal{N} \mathbf{C}_{\mathrm{KLM}}$. As we have done in Section 3.1 for the calculi without CSO, the standard proof is by induction on the height of the derivation of a sequent $\Gamma(\Delta, \Delta)$ (see Lemma 13). However, the standard proof does not work in the case of a proof of $\Gamma, \neg(C \Rightarrow D),[A$ : $\Delta],[A: \Delta]$ where the rule ending the derivation is $(C S O)$ : 


$$
\begin{aligned}
& \Pi_{1}
\end{aligned}
$$

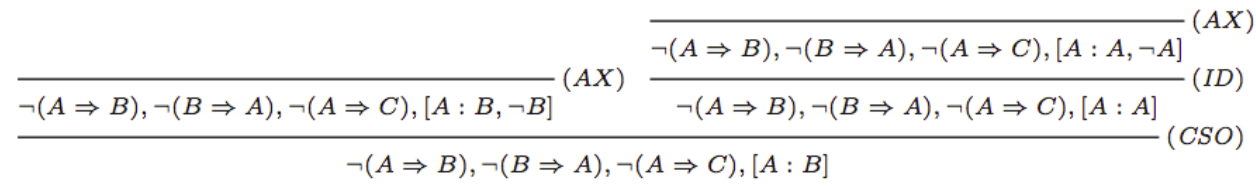

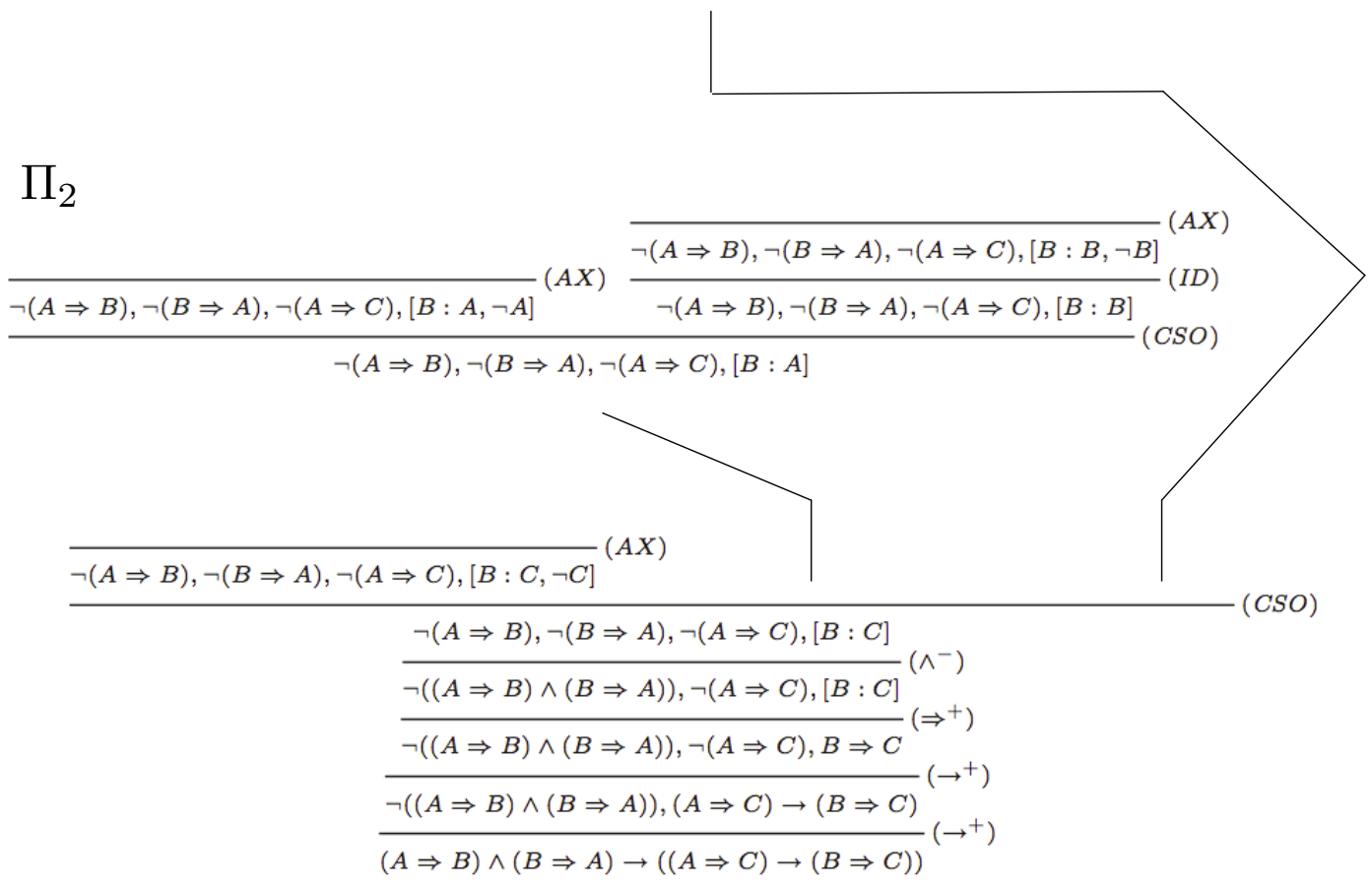

Figure 4: A derivation of the axiom CSO. Notice that the sequent $\neg(A \Rightarrow B), \neg(B \Rightarrow$ $A), \neg(A \Rightarrow C),[A: A]$ occurs twice as a premise of the application of the rule $(C S O)$ in the derivation $\Pi_{1}$; therefore, in order to increase readability, we only show it once. This is why the application of $(C S O)$ in $\Pi_{1}$ has two premises (and not three). The same for $\neg(A \Rightarrow B), \neg(B \Rightarrow A), \neg(A \Rightarrow C),[B: B]$, occurring twice in $\Pi_{2}$. 


$$
\frac{\neg(A \Rightarrow B), \neg(A \Rightarrow C),[A \wedge B: C, \neg C] \frac{\frac{\Sigma,[A \wedge B: A, \neg A, \neg B)]}{\Sigma,[A \wedge B: A, \neg(A \wedge B)]}\left(\wedge^{-}\right)}{\neg_{(A \Rightarrow B), \neg(A \Rightarrow C),[A \wedge B: A]}(I D)} \neg(A \Rightarrow B), \neg(A \Rightarrow C),[A: A \wedge B]}{\frac{\neg(A \Rightarrow B), \neg(A \Rightarrow C),[A \wedge B: C]}{\neg(A \Rightarrow B), \neg(A \Rightarrow C),(A \wedge B) \Rightarrow C}\left(\Pi_{1}\right)}
$$

where $\Pi_{1}$ is the following derivation:

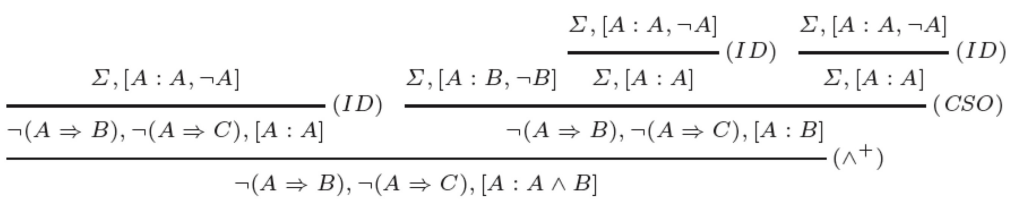

Figure 5: A derivation of axiom $\mathrm{CM}((A \Rightarrow B) \wedge(A \Rightarrow C)) \rightarrow(A \wedge B) \Rightarrow C$. We omit the first propositional steps, and we let $\Sigma=\neg(A \Rightarrow B), \neg(A \Rightarrow C)$.

$$
\begin{aligned}
\Gamma, \neg(C \Rightarrow D),[A: \Delta, \neg D],[A: \Delta] \\
\Gamma, \neg(C \Rightarrow D),[A: \Delta],[A: C] \\
\Gamma, \neg(C \Rightarrow D),[A: \Delta],[C: A] \\
\quad \Gamma, \neg(C \Rightarrow D),[A: \Delta],[A: \Delta]
\end{aligned}
$$

In order to obtain a derivation of $\Gamma, \neg(C \Rightarrow D),[A: \Delta]$, it is tempting to apply the inductive hypothesis to the leftmost premise ${ }^{6}$, obtaining a derivation of $\Gamma, \neg(C \Rightarrow$ $D),[A: \Delta, \neg D]$. However, the inductive hypothesis is not applicable to the other two premises, since they do not contain two copies of the same formula. Moreover, our objective would be to obtain derivations of $\Gamma, \neg(C \Rightarrow D),[A: C]$ and $\Gamma, \neg(C \Rightarrow$ $D),[C: A]$, but there is no way to remove $[A: \Delta]$ from the initial premises. Therefore, we are not able to get a derivation of $\Gamma, \neg(C \Rightarrow D),[A: \Delta]$ by applying $(C S O)$.

The proof of contraction in $\mathcal{N} \mathrm{C}_{\mathrm{KLM}}$ relies on a kind of disjunction property that holds only for a particular form of sequents, that do not contain other formulas than literals or negated conditionals. We call these sequents CSO-reduced sequents, and we define them in Definition 36:

Definition 36 (CSO-reduced sequent) $A$ sequent $\Gamma$ is CSO-reduced if it has the form $\Gamma=\Lambda, \Pi,\left[B_{1}: \Delta_{1}\right], \ldots,\left[B_{m}: \Delta_{m}\right]$, where $\Lambda$ is a multiset of literals and $\Pi$ is a multiset of negative conditionals.

The following proposition is a kind of disjunctive property for CSO-reduced sequents.

Proposition 37 Let $\Gamma=\Lambda, \Pi,\left[B_{1}: \Delta_{1}\right], \ldots,\left[B_{m}: \Delta_{m}\right]$ be CSO-reduced, if $\Gamma$ is derivable then for some $i$, the sequent $\Lambda, \Pi,\left[B_{i}: \Delta_{i}\right]$ is (height-preserving) derivable.

\footnotetext{
${ }^{6}$ We first obtain a derivation of $\Gamma, \neg(C \Rightarrow D),[A: \Delta, \neg D],[A: \Delta, \neg D]$ since weakening is heightpreserving admissible, then we apply the inductive hypothesis.
} 
Proof. By induction on the height of the derivation of $\Gamma$ : if $\Gamma$ is an axiom then the claim is easy and left to the reader. If $\Gamma$ is derived by any rule $(\mathbf{R})$ applied to some $\left[B_{j}: \Delta_{j}\right]$, we proceed as follows. We illustrate the most difficult case of $(C S O)$, the other are similar, but simpler. Thus suppose that $\Gamma$ is derived by (CSO) applied to $\neg(C \Rightarrow D)$ and $\left[B_{j}: \Delta_{j}\right]$, say $j=1$ to keep indexing easy, then $\Gamma=\Lambda, \Pi^{\prime}, \neg(C \Rightarrow D),\left[B_{1}: \Delta_{1}\right], \ldots,\left[B_{m}: \Delta_{m}\right]$ and the following are derivable with smaller height:

(a) $\Lambda, \Pi^{\prime}, \neg(C \Rightarrow D),\left[B_{1}: \Delta_{1}, \neg D\right],\left[B_{2}: \Delta_{2}\right], \ldots,\left[B_{m}: \Delta_{m}\right]$

(b) $\Lambda, \Pi^{\prime}, \neg(C \Rightarrow D),\left[B_{1}: C\right],\left[B_{2}: \Delta_{2}\right], \ldots,\left[B_{m}: \Delta_{m}\right]$

(c) $\Lambda, \Pi^{\prime}, \neg(C \Rightarrow D),\left[C: B_{1}\right],\left[B_{2}: \Delta_{2}\right], \ldots,\left[B_{m}: \Delta_{m}\right]$.

We can apply the inductive hypothesis to (a), (b), and (c), that is to say

- for (a), either $\left(a^{\prime}\right) \Lambda, \Pi^{\prime}, \neg(C \Rightarrow D),\left[B_{1}: \Delta_{1}, \neg D\right]$ is derivable or, for some $i,\left(a_{i}\right) \Lambda, \Pi^{\prime}, \neg(C \Rightarrow D),\left[B_{i}: \Delta_{i}\right]$ is derivable;

- for (b), either $\left(b^{\prime}\right) \Lambda, \Pi^{\prime}, \neg(C \Rightarrow D),\left[B_{1}: C\right]$ is derivable or, for some $j,\left(b_{j}\right) \Lambda, \Pi^{\prime}, \neg(C \Rightarrow D),\left[B_{j}: \Delta_{j}\right]$

- for (c), either $\left(c^{\prime}\right) \Lambda, \Pi^{\prime}, \neg(C \Rightarrow D),\left[C: B_{1}\right]$ is derivable or, for some

$k,\left(c_{k}\right) \Lambda, \Pi^{\prime}, \neg(C \Rightarrow D),\left[B_{k}: \Delta_{k}\right]$.

If for (a) we have that $\left(a_{i}\right)$ is derivable, then we are done. If for (b) we have that $\left(b_{j}\right)$ is derivable, then we are done too. The same if for (c) we have that $\left(c_{k}\right)$ is derivable. Otherwise, the following are derivable (with no greater height):

(a') $\Lambda, \Pi^{\prime}, \neg(C \Rightarrow D),\left[B_{1}: \Delta_{1}, \neg D\right]$

(b') $\Lambda, \Pi^{\prime}, \neg(C \Rightarrow D),\left[B_{1}: C\right]$

(c') $\Lambda, \Pi^{\prime}, \neg(C \Rightarrow D),\left[C: B_{1}\right]$.

By applying the $(C S O)$ rule we derive: $\Lambda, \Pi^{\prime}, \neg(C \Rightarrow D),\left[B_{1}: \Delta_{1}\right]$.

Proposition 38 Let $\Gamma=\Sigma,\left[B_{1}: \Delta_{1}\right], \ldots,\left[B_{m}: \Delta_{m}\right]$ be any sequent. If $\Gamma$ is derivable with a derivation of height $h$, then it can be derived from a set of derivable CSO-reduced sequents $\Gamma_{i}=\Sigma_{j},\left[B_{1}: \Delta_{1}\right], \ldots,\left[B_{m}: \Delta_{m}\right]$ with a derivation of height $h^{\prime} \leq h$. Moreover, all rules applied to derive $\Gamma$ from $\Gamma_{i}$ are either propositional rules or the rule $\left(\Rightarrow^{+}\right)$.

Proposition 38 can be proved by permuting (downwards) all the applications of propositional and $\left(\Rightarrow^{+}\right)$rules.

The following Proposition 39, which is based on Proposition 37 above, ensures the admissibility of contraction for $\mathcal{N} \mathbf{C}_{\mathrm{KLM}}$ in the critical case where the contracted formula is a context $[A: \Delta]$. The same argument does not extend immediately to the full language with nested conditionals.

Proposition 39 Let $\Gamma=\Sigma,[A: \Delta],[A: \Delta]$ be derivable, then $\Gamma=\Sigma,[A: \Delta]$ is (height-preserving) derivable.

Proof. By Proposition 38, $\Gamma$ is height-preserving derivable from a set of CSO-reduced sequents $\Sigma_{i},[A: \Delta],[A: \Delta]$. By Proposition 37 , each $\Sigma_{i},[A: \Delta]$ is derivable; we then obtain $\Sigma,[A: \Delta]$ by applying the same sequence of rules. 
Lemma 40 (Admissibility of contraction in $\mathcal{N C}_{\mathbf{K L M}}$ ) Contraction is admissible in $\mathcal{N} \mathbf{C}_{K L M}$ : given a nested sequent $\Sigma$, if $\Gamma(\Sigma, \Sigma)$ is derivable, then $\Gamma(\Sigma)$ is (heightpreserving) derivable.

Proof. If $\Sigma$ is a formula the proof is exactly the same as the one for Lemma 13 in $\mathcal{N} S$, except for the following case of $(C S O)$ : if $\Sigma=\neg(C \Rightarrow D)$, and the derivation is ended as follows:

$$
\begin{aligned}
& \Gamma, \neg(C \Rightarrow D), \neg(C \Rightarrow D),[A: \Delta, \neg D] \\
& \Gamma, \neg(C \Rightarrow D), \neg(C \Rightarrow D),[A: C] \\
& \Gamma, \neg(C \Rightarrow D), \neg(C \Rightarrow D),[C: A] \\
& \hline \Gamma, \neg(C \Rightarrow D), \neg(C \Rightarrow D),[A: \Delta]
\end{aligned}(C S O)
$$

we apply the inductive hypothesis on the three premises, obtaining derivations of

(i) $\Gamma, \neg(C \Rightarrow D),[A: \Delta, \neg D]$

(ii) $\Gamma, \neg(C \Rightarrow D), \neg(C \Rightarrow D),[A: C]$

(iii) $\Gamma, \neg(C \Rightarrow D),[C: A]$.

We obtain a derivation of $\Gamma, \neg(C \Rightarrow D),[A: \Delta]$ by applying $(C S O)$ to $(i),(i i)$ and (iii).

If $\Sigma=[A: \Delta]$ we conclude by applying Proposition 39 .

As usual, we obtain completeness by cut-elimination. As in case of $\mathcal{N} S$, the proof is by mutual induction together with a substitution property:

Theorem 41 (Admissibility of cut in $\mathcal{N C}_{\mathrm{KLM}}$ ) In $\mathcal{N} \mathrm{C}_{\mathrm{KLM}}$, the following propositions hold:

- (A) If $\Gamma(F)$ and $\Gamma(\neg F)$ are derivable, then so is $\Gamma$;

- (B) if $(I) \Gamma,[A: \Delta],(I I) \Gamma,\left[A: A^{\prime}\right]$, and $(I I I) \Gamma,\left[A^{\prime}: A\right]$ are derivable, then so is $\Gamma,\left[A^{\prime}: \Delta\right]$.

Proof. To make the structure of the induction clear call: $C u t(c, h)$ the property (A) for any $\Gamma$ and any formula $F$ of complexity $c$ and such that the sum of the heights of derivation of the premises is $h$. Similarly call $S u b(c)$ the assertion that (B) holds for any $\Gamma$ and any formula $A$ of complexity $c$. The proof of both is by mutual induction on the complexity $c$. We show the following facts:

(A) $\forall c \forall h\left(\left(\forall c^{\prime}<c \forall h^{\prime} C u t\left(c^{\prime}, h^{\prime}\right) \wedge \forall h^{\prime \prime}<h C u t\left(c, h^{\prime \prime}\right)\right) \rightarrow C u t(c, h)\right)$

(B) $\forall c(\forall h C u t(c, h) \rightarrow \operatorname{Sub}(c))$

This will prove that $\forall c \forall h C u t(c, h)$ and $\forall c S u b(c)$. The proof of (A) is the same as the one of Theorem 16, except in the following cases in which the principal formula is introduced by $(C S O)$. We have two cases: (d') in which the cut formula is introduced by $(C S O)-\left(\Rightarrow^{+}\right)$and $\left(\mathrm{e}^{\prime}\right)$ in which the cut formula is introduced by $(C S O)-(I D)$.

- (d') the derivation is as follows:

$$
\begin{aligned}
& \text { (1) } \Gamma,[A: \Delta, \neg D], \neg(C \Rightarrow D) \\
& \text { (2) } \Gamma,[A: C], \neg(C \Rightarrow D)
\end{aligned}
$$

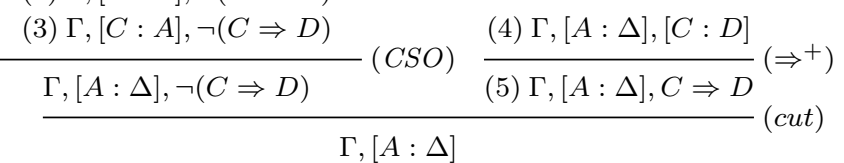


First of all, since weakening is admissible (Lemma 34), from (5) we obtain a derivation, of no greater height, also for $\left(5^{\prime}\right) \Gamma,[A: \Delta, \neg D], C \Rightarrow D$. We then apply the inductive hypothesis on (A) to cut $(1)$ and $\left(5^{\prime}\right)$, to obtain a derivation of $(6) \Gamma,[A: \Delta, \neg D]$, from which we obtain a derivation of $\left(6^{\prime}\right) \Gamma,[A: \Delta],[A:$ $\Delta, \neg D]$ by weakening. Since we have derivations for $(2)$ and $(3)$, we can also apply the inductive hypothesis on (B), that is to say $\forall c^{\prime}<c S u b\left(c^{\prime}\right)$, to obtain a derivation of $\left(4^{\prime}\right) \Gamma,[A: \Delta],[A: D]$ from (4), from which we obtain a derivation of $\left(4^{\prime \prime}\right) \Gamma,[A: \Delta],[A: \Delta, D]$ by weakening. We conclude by applying the inductive hypothesis for $(\mathrm{A})$ on the complexity of the cut formula to cut $\left(6^{\prime}\right)$ and $\left(4^{\prime \prime}\right)$ : we obtain a derivation of $\Gamma,[A: \Delta],[A: \Delta]$, from which we conclude that $\Gamma,[A: \Delta]$ is derivable since contraction is admissible (Lemma $40)$

- (e') the derivation is as follows:

$$
\begin{aligned}
& \Gamma,[A: \Delta, F, \neg D], \neg(C \Rightarrow D) \\
& \Gamma,[A: C], \neg(C \Rightarrow D) \\
& \frac{\Gamma,[C: A], \neg(C \Rightarrow D)}{(1) \Gamma, \neg(C \Rightarrow D),[A: \Delta, F]}(C S O) \frac{(2) \Gamma, \neg(C \Rightarrow D),[A: \Delta, \neg F, \neg A]}{\Gamma, \neg(C \Rightarrow D),[A: \Delta, \neg F]}(\text { cut })
\end{aligned}
$$

First of all, since weakening is admissible (Lemma 34), from (1) we obtain a derivation, of no greater height, also of $\left(1^{\prime}\right) \Gamma, \neg(C \Rightarrow D),[A: \Delta, F, \neg A]$. We can apply the inductive hypothesis on (A) to cut $\left(1^{\prime}\right)$ and (2) (on the sum of the heights of the derivations), then we obtain a derivation of $\Gamma, \neg(C \Rightarrow D),[A$ : $\Delta, \neg A]$, to which we apply $(I D)$ to obtain a derivation of $\Gamma, \neg(C \Rightarrow D),[A: \Delta]$, and we are done.

To prove (B), we first introduce the notion of rank: the rank $r$ of an instance of (B) is the 3-multiset $r=\left(h_{1}, h_{2}, h_{3}\right)$ of the respective heights of the premises of (B): $h_{1}$ is the height of $\Gamma([A: \Delta]), h_{2}$ of $\Gamma\left(\left[A: A^{\prime}\right]\right)$, and $h_{3}$ of $\Gamma\left(\left[A^{\prime}: A\right]\right)$. Given two ranks $r=\left(h_{1}, h_{2}, h_{3}\right)$ and $r^{\prime}=\left(h_{1}^{\prime}, h_{2}^{\prime}, h_{3}^{\prime}\right)$ we define the following ordering (namely a case of multi-set ordering): $r<r^{\prime}$ iff $r$ is obtained by replacing at least one $h_{j}^{\prime}$ with 1 to 3 strictly smaller values $h_{i}$. This relation is transitive and well-founded, so that we will use it to prove statement (B) by induction on the rank $r$, where we suppose that the complexity of formula $A$ is $c$. All cases are easy except when $\Gamma([A: \Delta])$ is derived by $(C S O)$ and by $(I D)$.

- $(C S O)$. The derivation is as follows:

$$
\frac{(1) \Gamma, \neg(C \Rightarrow D),[A: \Delta, \neg D] \quad(2) \Gamma, \neg(C \Rightarrow D),[A: C] \quad(3) \Gamma, \neg(C \Rightarrow D),[C: A]}{\Gamma, \neg(C \Rightarrow D),[A: \Delta]}(C S O)
$$

Let $h_{1}$ be the height of the conclusion, the two other hypothesis are: (4) $\Gamma, \neg(C \Rightarrow$ $D),\left[A: A^{\prime}\right]$ with height $h_{2}$ and $(5) \Gamma, \neg(C \Rightarrow D),\left[A^{\prime}: A\right]$ with height $h_{3}$, so that the instance of (B) with $\Gamma, \neg(C \Rightarrow D),[A: \Delta]$, (4), (5) has rank $r=\left(h_{1}, h_{2}, h_{3}\right)$. Let $k_{1}, k_{2}, k_{3}<h_{1}$ be the heights of the three premises (1), (2), (3) in that order. We have that an instance of (B) with premise (1), (4), (5) has rank $\left(k_{1}, h_{2}, h_{3}\right)<r$ and the instance of (B) with (2), (4), (5) has rank $\left(k_{2}, h_{2}, h_{3}\right)<r$. By inductive hypothesis we obtain: 


$$
\begin{gathered}
\left(1^{\prime}\right) \Gamma, \neg(C \Rightarrow D),\left[A^{\prime}: \Delta, \neg D\right] \\
\quad\left(2^{\prime}\right) \Gamma, \neg(C \Rightarrow D),\left[A^{\prime}: C\right] .
\end{gathered}
$$

Moreover an instance of (B) with (4), (2) and (3) has rank $\left(h_{2}, k_{2}, k_{3}\right)<$ $\left(h_{1}, h_{2}, h_{3}\right)=r$, so that we can apply the induction hypothesis and get

$$
\left(4^{\prime}\right) \Gamma, \neg(C \Rightarrow D),\left[C: A^{\prime}\right] .
$$

Now we apply $(C S O)$ to (1'), (2'), (4') and we obtain $\Gamma, \neg(C \Rightarrow D),\left[A^{\prime}: \Delta\right]$.

- $(I D)$ In this case, we have

$$
\frac{(1) \Gamma,[A: \Delta, \neg A]}{\Gamma,[A: \Delta]}(I D)
$$

The height of the premise (1), say $h_{1}^{\prime}$, is smaller than $h_{1}$ the height of the conclusion, so that $\left(h_{1}^{\prime}, h_{2}, h_{3}\right)<\left(h_{1}, h_{2}, h_{3}\right)$, thus by inductive hypothesis we get:

$$
\text { (6) } \Gamma,\left[A^{\prime}: \Delta, \neg A\right] \text {. }
$$

From the hypothesis of (B) that $\Gamma,\left[A^{\prime}: A\right]$ is derivable, we get by weakening:

$$
\text { (7) } \Gamma,\left[A^{\prime}: \Delta, A\right] \text {. }
$$

Since by hypothesis we have cut on a formula of complexity $c$, by cutting (6) and (7) we obtain $\Gamma,\left[A^{\prime}: \Delta\right]$.

Theorem 42 The calculus $\mathcal{N} \mathbf{C}_{K L M}$ is sound and complete for KLM logic $\mathbf{C}$ (corresponding to the flat fragment of $\mathrm{CK}+\mathrm{CSO}+\mathrm{ID})$.

Proof. For soundness we have just to check the validity of the $(C S O)$ rule. In this case, $\Gamma, \neg(C \Rightarrow D),[A: \Delta]$ is derived from $(i) \Gamma, \neg(C \Rightarrow D),[A: \Delta, \neg D]$, (ii) $\Gamma, \neg(C \Rightarrow D),[A: C]$, and (iii) $\Gamma, \neg(C \Rightarrow D),[C: A]$. We show that $(*)(\neg(C \Rightarrow D) \vee(A \Rightarrow(\Delta \vee \neg D))) \wedge(\neg(C \Rightarrow D) \vee(A \Rightarrow C)) \wedge(\neg(C \Rightarrow D) \vee(C \Rightarrow A)) \rightarrow$ $\rightarrow(\neg(C \Rightarrow D) \vee(A \Rightarrow \Delta))$

is valid in $\mathrm{CK}+\mathrm{CSO}+\mathrm{ID}$, then we conclude by propositional reasoning and the inductive hypothesis. The mentioned formula is derivable as follows: by (RCK) we have

$$
\text { (1) }(A \Rightarrow D) \wedge(A \Rightarrow(\Delta \vee \neg D)) \rightarrow(A \Rightarrow \Delta)
$$

By (CSO) we have:

$$
\text { (2) }(A \Rightarrow C) \wedge(C \Rightarrow A) \wedge(C \Rightarrow D) \rightarrow(A \Rightarrow D)
$$

Thus by (1) and (2) we get:

$$
(3)(A \Rightarrow C) \wedge(C \Rightarrow A) \wedge(C \Rightarrow D) \wedge(A \Rightarrow(\Delta \vee \neg D)) \rightarrow(A \Rightarrow \Delta)
$$


Let us abbreviate by $F$ the premise of (3), by propositional reasoning we get that:

$$
(\neg(C \Rightarrow D) \vee F) \rightarrow(\neg(C \Rightarrow D) \vee(A \Rightarrow \Delta))
$$

But then we get (*) by distributing $\neg(C \Rightarrow D)$ over the conjuncts of $F$ and obvious simplification.

For completeness, one can derive all instances of CSO axioms as shown in Figure 4. Moreover the rules RCK and RCEA are derivable too, as follows.

For (RCEA), we have to show that if $A \leftrightarrow B$ is derivable, then also $(A \Rightarrow C) \leftrightarrow(B \Rightarrow$ $C)$ is derivable. Since $A \leftrightarrow B$ is derivable, and since $\left(\wedge^{+}\right)$and $\left(\rightarrow^{+}\right)$are invertible, we have a derivation for $A \rightarrow B$, then for $(1) \neg A, B$, and for $B \rightarrow A$, then for (2) $A, \neg B$; therefore, by the generalization of weakening of Lemma 35, we have derivations for $\left(1^{\prime}\right)[A: \neg A, B]$, and for $\left(2^{\prime}\right)[B: A, \neg B]$. We derive $(A \Rightarrow C) \rightarrow(B \Rightarrow C)$ (the other half is symmetric) as follows:

$$
\begin{gathered}
\frac{\neg(A \Rightarrow C),[B: C, \neg C]}{(A X) \frac{\left(1^{\prime}\right)[A: \neg A, B]}{[A: B]}(I D) \frac{\left(2^{\prime}\right)[B: A, \neg B]}{[B: A]}(I D)}(C S O) \\
\frac{\neg(A \Rightarrow C),[B: C]}{\frac{\neg(A \Rightarrow C), B \Rightarrow C}{(A \Rightarrow C) \rightarrow(B \Rightarrow C)}\left(\rightarrow^{+}\right)}
\end{gathered}
$$

For $(\mathrm{RCK})$, suppose that we have a derivation of $\left(A_{1} \wedge \ldots \wedge A_{n}\right) \rightarrow B$. Since $\left(\rightarrow^{+}\right)$ is invertible, we have also a derivation of $B, \neg\left(A_{1} \wedge \ldots \wedge A_{n}\right)$. Since $\left(\wedge^{-}\right)$is also invertible, then we have a derivation of $B, \neg A_{1}, \ldots, \neg A_{n}$ and, again by Lemma 35 , of $(1) \neg\left(C \Rightarrow A_{1}\right), \ldots, \neg\left(C \Rightarrow A_{n}\right),\left[C: B, \neg A_{1}, \neg A_{2}, \ldots, \neg A_{n}\right]$, from which we conclude as follows:

(1) $\neg\left(C \Rightarrow A_{1}\right), \ldots, \neg\left(C \Rightarrow A_{n}\right),\left[C: B, \neg A_{1}, \neg A_{2}, \ldots, \neg A_{n}\right]$

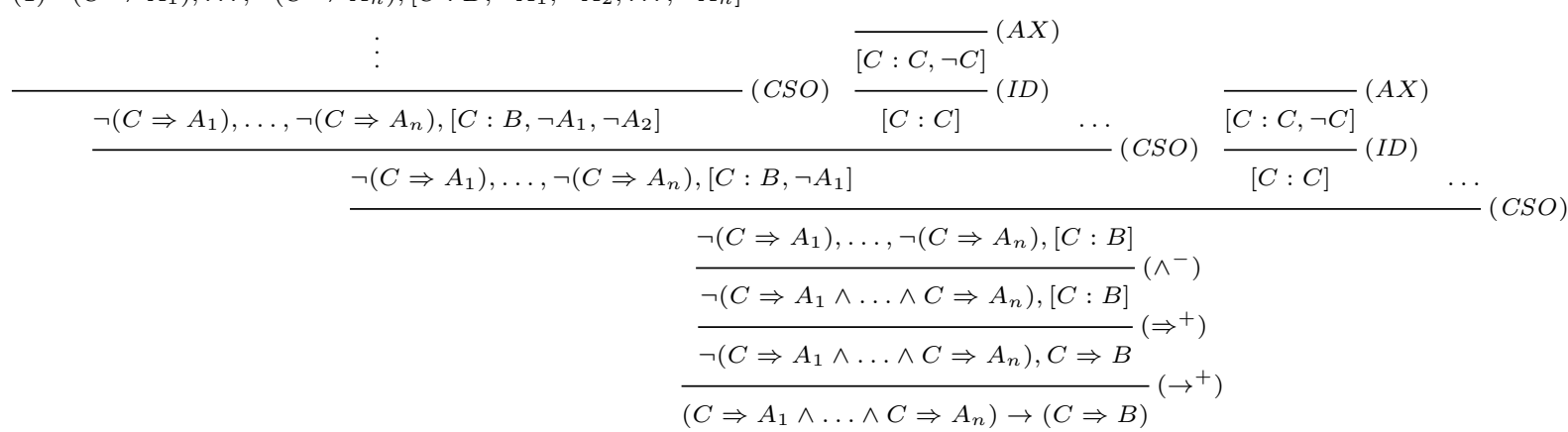

For closure under (Modus Ponens), as usual we use the previous Theorem 41 exactly as we have done to show that this rule is derivable in the calculus $\mathcal{N} S$ in the proof of Theorem 18.

\subsection{Termination and complexity of $\mathcal{N} \mathrm{C}_{\mathrm{KLM}}$}

Termination of the calculus $\mathcal{N} \mathbf{C}_{\mathrm{KLM}}$ can be proved similarly to Theorem 20: in this case, the rules $(C S O)$ and $(I D)$ can be applied infinitely often, however we can control the application of these rules in order to obtain a terminating calculus. For $(I D)$, the 
restriction is exactly the same adopted for $\mathcal{N} S$, namely it is applied only once to each context $[A: \Delta]$ in each branch. As mentioned, the rule $(C S O)$ in $\mathcal{N} \mathbf{C}_{\mathrm{KLM}}$ replaces the rule $\left(\Rightarrow^{-}\right)$of $\mathcal{N} S$ : for this rule, we show that we can impose a restriction similar to the one imposed on $\left(\Rightarrow^{-}\right)$in $\mathcal{N} S$, that is to say $(C S O)$ is applied only once to each formula $\neg(A \Rightarrow B)$ with a context $\left[A^{\prime}: \Delta\right]$ in each branch.

In order to obtain a terminating calculus, we put the following restrictions on the application of $(C S O)$ and $(I D)$ :

Restrictions for terminating $\mathcal{N} \mathrm{C}_{K L M}$.

- apply $(C S O)$ to $\Gamma, \neg(A \Rightarrow B),\left[A^{\prime}: \Delta\right]$ only if $(C S O)$ has not been applied to the formula $\neg(A \Rightarrow B)$ with the context $\left[A^{\prime}: \Delta\right]$ in the current branch;

- apply $(I D)$ to $\Gamma([A: \Delta])$ only if $(I D)$ has not been applied to $[A: \Delta]$ in the current branch.

Theorem 43 The calculus $\mathcal{N} \mathbf{C}_{K L M}$ with the termination restrictions is sound and complete for KLM logic $\mathbf{C}$ (corresponding to the flat fragment of $\mathrm{CK}+\mathrm{CSO}+\mathrm{ID}$ ).

Proof. We show that it is useless to apply the rules $(C S O)$ and $(I D)$ without the restrictions.

- $(C S O)$ : suppose it is applied twice on $\Gamma, \neg(A \Rightarrow B),\left[A^{\prime}: \Delta\right]$ in a branch. Since $(C S O)$ is "weakly" invertible, we can assume, without loss of generality, that the two applications of $(C S O)$ are consecutive, starting from $\Gamma, \neg(A \Rightarrow$ $B),\left[A^{\prime}: \Delta, \neg B, \neg B\right]$. By Lemma 40 (contraction), we have a derivation of $\Gamma, \neg(A \Rightarrow B),\left[A^{\prime}: \Delta, \neg B\right]$, and we can conclude with a (single) application of $(C S O)$. Remember that contraction is rule-preserving admissible, therefore the obtained derivation does not add any application of $(C S O)$;

- $(I D)$ : the same proof as in Theorem 6.

The above restrictions ensure a terminating proof search for the systems under consideration, in particular:

Theorem 44 The calculus $\mathcal{N} \mathbf{C}_{K L M}$ with the termination restrictions give a PSPACE decision procedure for KLM logic $\mathbf{C}$ (corresponding to the flat fragment of $\mathrm{CK}+\mathrm{CSO}+\mathrm{ID}$ ).

Proof. We proceed as we have done in the proof of Theorem 20 for proving termination of $\mathcal{N} S$, namely we give bounds to the size of a sequent, and to the size of a derivation. In order to do that, we first give a bound to the number of contexts that can appear in a sequent occurring in a derivation.

Let $\Pi$ be a derivation of a sequent $\Gamma$ in $\mathcal{N} \mathbf{C}_{\mathrm{KLM}}$, and $\Lambda$ be a sequent occurring in $\Pi$. Since $\mathcal{N} \mathbf{C}_{\mathrm{KLM}}$ takes into account only the flat fragment, i.e. without nested conditionals, of the logic $\mathrm{CK}+\mathrm{CSO}+\mathrm{ID}$, we can easily observe that, given a context $[A: \Delta]$ occurring in a sequent, $\Delta$ only contains propositional formulas; in other words, as a difference with $\mathcal{N} S$, contexts are no longer nested. Since contexts are only created by the rule $\left(\Rightarrow^{+}\right)$, the maximum number of contexts that can appear in $\Lambda$ in $\Pi$ is in 
$\mathrm{O}(|\Gamma|)$. Furthermore, given a context $[A: \Delta]$, by the termination restrictions we have that $|\Delta|$ is linearly bounded by $|\Gamma|$.

We also have to take into account the extra space that may be needed to implement the termination restrictions. Exactly as we have done in the proof of Lemma 23 for $\mathcal{N} S$, we can observe that these restrictions can be implemented by some bookkeeping mechanism, that is for each context we store the list of (negative conditional) formulas to which the rule $(C S O)$ has been applied with this context. Since this rule can only be applied once to each formula with respect to a given context, the extra space overhead is in $\mathrm{O}(|\Gamma|)$. For the rule $(I D)$, we can simply use a flag to record whether the rule has been applied to a given context conditional. This gives a constant space overhead for each context. Therefore, the space needed to store a single context (taking the termination restrictions into account) is in $\mathrm{O}(|\Gamma|)$. The number of contexts that can appear in $\Lambda$ being in $\mathrm{O}(|\Gamma|)$, we conclude that $|\Lambda| \in \mathrm{O}\left(|\Gamma|^{2}\right)$.

We now bound the length of a branch in a derivation. First, observe that the rules $\left(\Rightarrow^{+}\right)$and $(C S O)$ can only be applied to conditional formulas appearing in $\Lambda$ (contexts only contain propositional formulas). Hence, the number of applications of $\left(\Rightarrow^{+}\right)$is linearly bounded by $|\Gamma|$. Since this is the only rule which can introduce new contexts, this also gives a (linear) bound to the number of contexts that can appear in $\Delta$. Due to the termination restrictions, the rule $(C S O)$ can be applied once for each negative conditional formula and for each context occurring in $\Lambda$. Both numbers being linearly bounded by $|\Gamma|$, the number of applications of the rule $(C S O)$ is linearly bounded by $|\Gamma|^{2}$. Since the number of contexts in $\Lambda$ is bounded by $|\Lambda|$, and since $(I D)$ can be applied only once to each context, there is at most $\mathrm{O}(|\Gamma|)$ applications of $(I D)$.

Let $[A: \Delta]$ be a context occurring in $\Lambda$. We estimate the number of rules that can be applied within this context, i.e. the number of rules that can be applied to formulas of $\Delta$. We observe that: 1. As usual, the boolean rules cannot be applied redundantly and the number of applications of these rules is linearly bounded by the number of boolean subformulas occurring in $\Delta$, which is itself linearly bounded by $|\Gamma|$. 2. The rules $\left(\Rightarrow^{+}\right),(C S O)$, and $(I D)$ can no longer be applied to conditional formulas appearing in subformulas of $\Delta$, since $\Delta$ contains only propositional formulas.

Therefore, the number of rule applications in a given context is linearly bounded by $|\Gamma|$. The number of contexts that can appear in $\Lambda$ being bounded by $|\Gamma|$, we easily obtain the linear bound of $|\Gamma|^{2}$ to the number of applications of rules in any branch of the derivation.

By the bounds on the size of a sequent and the number of rule applications in a given branch of a derivation, we conclude that the size of each branch is in $\mathrm{O}\left(|\Gamma|^{4}\right)$.

We do not know whether this bound is optimal. The study of the optimal complexity for the flat fragment of $\mathrm{CK}+\mathrm{CSO}+\mathrm{ID}$ is still open. A NEXP tableau calculus for cumulative logic $\mathbf{C}$ has been proposed in [17]. In [30] the authors provide calculi for full (i.e. with nested conditionals) $\mathrm{CK}+\mathrm{ID}+\mathrm{CM}$ and $\mathrm{CK}+\mathrm{ID}+\mathrm{CM}+\mathrm{CA}$ : these logics are related to $\mathrm{CK}+\mathrm{ID}+\mathrm{CSO}$, but they do not coincide with it ${ }^{7}$, even for the flat fragment, i.e. cumulative logic $\mathbf{C}$ as $\mathrm{CSO}=\mathrm{CM}+\mathrm{RT}$ (restricted transitivity). Their calculi are internal, but rather complex as they make use of ingenious yet highly combinatorial rules. They obtain a PSPACE bound in all cases.

\footnotetext{
${ }^{7}$ In [11] it is shown that Kraus, Lehmann and Magidor's preferential logic $\mathbf{P}$ corresponds to the flat fragment of the conditional logic $\mathrm{CK}+\mathrm{ID}+\mathrm{AC}+\mathrm{CA}+\mathrm{RT}$, the last one equivalent to $\mathrm{CK}+\mathrm{CSO}+\mathrm{ID}+\mathrm{CA}$.
} 


\section{Further Work}

\section{Completing the cube of extensions of CK}

In this work we have provided nested sequent calculi for the basic normal conditional logic CK and its extensions with ID, MP, and CEM, namely all combinations except $\mathrm{CK}+\mathrm{MP}+\mathrm{CEM}(+\mathrm{ID})$. It is easy to see that the cut-free calculus $\mathcal{N} S$ combining the rules $(M P)$ and $(C E M)$ is not complete for system $\mathrm{CK}+\mathrm{MP}+\mathrm{CEM}$ : the sequent

$$
\neg A, \neg B,[A: B]
$$

admits a derivation with cut:

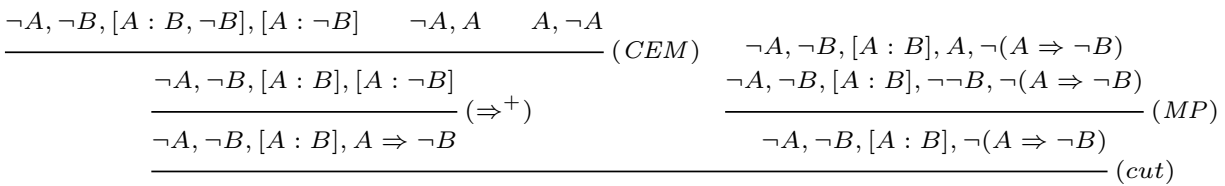

$$
\begin{aligned}
& \neg A, \neg B,[A: B]
\end{aligned}
$$

On the other hand, the sequent $\neg A, \neg B,[A: B]$ does not admit a cut-free proof, since no rule of $\mathrm{CK}+\mathrm{MP}+\mathrm{CEM}$ is backwards applicable to it (for arbitrary $A$ and $B$, say atoms).

Notice that this sequent correspnds to the formula $\neg A \vee \neg B \vee(A \Rightarrow B)$, which in turn it is equivaent to:

$$
\text { (CS) } \quad(A \wedge B) \rightarrow(A \Rightarrow B)
$$

This is nothing else than the axiom of Conditional Strong Centering (CS). We leave to the reader to check (easily) that this axiom is derivable in $\mathrm{CK}+\mathrm{CEM}+\mathrm{MP}^{8}$. A rule capturing (CS) is the following:

$$
\frac{\Gamma([C: \Delta], C) \quad \Gamma([C: \Delta], \Delta)}{\Gamma([C: \Delta])}(C S)
$$

By using this rule we get immediatly a derivation of the above sequent $\neg A, \neg B,[A: B]$, whence of any instance of (CS), as follows:

$$
\begin{gathered}
\frac{\neg A, \neg B,[A: B], A \quad \neg A, \neg B,[A: B], B}{(C S)} \\
\frac{\neg A, \neg B,[A: B]}{\neg A, \neg B, A \Rightarrow B}\left(\Rightarrow^{+}\right) \\
\frac{\neg(A \wedge B), A \Rightarrow B}{(A \wedge B) \rightarrow(A \Rightarrow B)}\left(\wedge^{-}\right) \\
\left(\rightarrow^{+}\right)
\end{gathered}
$$

We strongly conjecture that adding this rule to the calculus for $\mathrm{CK}$ we obtain a cut-free calculus for $\mathrm{CK}+\mathrm{CS}$ and adding it to the calculus for $\mathrm{CK}+\mathrm{MP}+\mathrm{CEM}$ we get a cut-free calculus for this system. In other words, by adding this rule we obtain cut-free calculi

\footnotetext{
${ }^{8}$ It is worth noticing that the semantic condition corresponding to (CS), namely $w \in[A] \rightarrow f(w,[A]) \subseteq$ $\{w\}$ follows directly from the semantics conditions corresponding to (MP) and to (CEM), respectively $w \in[A] \rightarrow w \in f(w,[A])$ and $|f(w,[A])| \leq 1$.
} 
for the whole cube of the extensions of CK generated by axioms (ID), (MP), (CEM) and (CS). Unfortunately, proving the admissibility of cut for any system including the (CS) rule seems hard and will be object of subsequent research.

\section{Preferential extensions of CK}

We would like to provide nested sequent calculi for preferential conditional logics, as they still lack "simple" and internal calculi. In general, by preferential conditional logics we mean those conditional logics that are characterized by models based on preferential structures (equipped by a familly of order relations $<_{x}$ indexed on worlds). In these models the selection function $f(A, x)$ is defined as the set of minimal- $A$ worlds with respect to the relation $<_{x}$. Whenever the order relations are modular, these models become equivalent to the so-called sphere models, introduced by Lewis, so that the family of logics defined by this semantics covers all conditional logics defined by Lewis. These logics satisfy the axiom (CV):

$$
(\mathrm{CV}) \quad(A \Rightarrow C) \wedge \neg(A \Rightarrow \neg B) \rightarrow(A \wedge B \Rightarrow C)
$$

This axiom is related to rational monotonicity of Lehmann and Magidor's rational logic $\mathbf{R}$ [23].

A first step in this direction is the calculus for the flat version of $\mathrm{CK}+\mathrm{ID}+\mathrm{CSO}$, corresponding to KLM logic of cumulativity $\mathbf{C}$ and characterized by cumulative models, we conjecture that it can be extended to whole $\mathrm{CK}+\mathrm{CSO}+\mathrm{ID}$, although the preferential semantics for the whole system is unknown. Since for preferential logics the semantics is given in terms of ordered models, it might turn out that the basic connective to consider is no longer the conditional operator, but a kind of entrenchment operator $A \triangleleft B$, whose meaning is " $A$ is more plausible than $B$ " (similarly to what is done in [24]) which reflects the ordered semantics more naturally. The conditional operator would then be obtained as a derived operator:

$$
A \Rightarrow B=(\perp \triangleleft A) \vee \neg((A \wedge \neg B) \triangleleft(A \wedge B))
$$

that is $A \Rightarrow B$ holds if $A$ is impossible or $A \wedge B$ is strictly more plausible than $A \wedge \neg B$.

\section{Efficiency and optimal procedures}

We also intend to study refinements of the calculi aiming at efficiency, based on a tighter control of formula duplication. The calculi we have presented in this work allows one to obtain a PSPACE upper bound for decidability in each logic. For some logics this bound is optimal, for those ones containing CEM it is not, since these logics are known to be in CONP. To this regard, we conjecture that the $(C E M)$ rule may be replaced by the following simpler one $\left(C E M^{\prime}\right)$ :

$$
\frac{\Gamma([A: \Delta, \Sigma]) \quad \neg A, B \quad \neg B, A}{\Gamma([A: \Delta],[B: \Sigma])}\left(C E M^{\prime}\right)
$$

If we replace the $(C E M)$ rule by $\left(C E M^{\prime}\right)$, it might possible to obtain a more efficient (optimal?) procedure for logics including CEM. Here is a rough idea for CK+CEM: the procedure would first apply $\left(C E M^{\prime}\right)$-rule backwards as much as possible to "merge" contexts. After "merging", for any pair of remaining contexts $[C: \Gamma],[D: \Lambda]$, the 
formulas $C$ and $D$ cannot be equivalent (otherwise the contexts would have been merged), whence there might be at most one application of the $\left(\Rightarrow^{-}\right)$rule for exactly one of such contexts; after the application of $\left(\Rightarrow^{-}\right)$, the involved negative conditional can be removed. Basing on this idea, we conjecture that we can obtain more efficient, possibly optimal, procedure, but the first step is to prove that we can safely replace $(C E M)$ by $\left(C E M^{\prime}\right)$.

\section{Further topics}

We wish to take advantage of the calculi to study logical properties of the corresponding systems (disjunction property, interpolation) in a constructive way. Another topic which deserves investigation is the precise relation of nested sequent calculi with other calculi for conditional logics, in particular with labelled calculi. Some labelled calculi for the conditional logics considered in this work have been presented in [28]. It would be interesting whether we can find a mapping between those calculi and nested sequent ones, as done by Fitting in his compresive work [12], where he establishes a tight correspondence between nested sequent calculi for modal logics and prefixed tableaux.

\section{Conclusions}

In this work we have provided nested sequent calculi for the basic normal conditional logic CK and a few extensions of it with combinations of ID, MP, and CEM, namely all combinations except $\mathrm{CK}+\mathrm{MP}+\mathrm{CEM}(+\mathrm{ID})$. The calculi are analytic and they enjoy a cut-elimination property. The calculi can be used to obtain a decision procedure, in some cases of optimal complexity. We have also provided a nested sequent calculus for cumulative logic $\mathbf{C}$ of the KLM framework corresponding to the flat fragment of $\mathrm{CK}+\mathrm{CSO}+\mathrm{ID}$ for which no internal calculus seem to be known so far.

Even if for some of the logics considered in this paper there exist other proof systems, we think that nested sequents provide internal calculi that are particularly natural and simple.

Obviously, it is our goal to extend them to a wider spectrum of conditional logics, first of all to preferential conditional logics, as they still lack "simple" and internal calculi.

We also intend to study refinements of the calculi aiming at efficiency. Finally, we wish to take advantage of the calculi to study logical properties of the corresponding systems (disjunction property, interpolation) in a constructive way.

\section{Acknowledgements}

We are grateful to the anonymous referees for their careful reading and constructive criticisms, which greatly helped us to improve the final version of our work.

Gian Luca Pozzato wants to dedicate this work to the memory of Fabio Varesano. Fabio was a young and promising researcher. He did not have enough time to realize all his dreams, but he had enough time to show a great passion for his research activity. 


\section{References}

[1] Alenda, R., Olivetti, N., Pozzato, G.L.: Nested Sequent Calculi for Conditional Logics. In: Luis Fariñas del Cerro, Andreas Herzig, J.M. (ed.) Logics in Artificial Intelligence - 13th European Conference, JELIA 2012. Lecture Notes in Artificial Intelligence (LNAI), vol. 7519, pp. 14-27. Springer-Verlag, Toulouse, France (Sptember 2012)

[2] Artosi, A., Governatori, G., Rotolo, A.: Labelled tableaux for non-monotonic reasoning: Cumulative consequence relations. Journal of Logic and Computation 12(6), 1027-1060 (2002)

[3] Baltag, A., Smets, S.: The logic of conditional doxastic actions. Texts in Logic and Games, Special Issue on New Perspectives on Games and Interaction 4, 9-31 (2008)

[4] Board, O.: Dynamic interactive epistemology. Games and Economic Behavior 49(1), 49-80 (2004)

[5] Boutilier, C.: Conditional logics of normality: a modal approach. Artificial Intelligence 68(1), 87-154 (1994)

[6] Brünnler, K.: Deep sequent systems for modal logic. Archive for Mathematical Logic 48(6), 551-577 (2009), http: //www.iam. unibe.ch/ kai/Papers/2009dssml.pdf

[7] Brünnler, K.: Nested sequents (2010), habilitation Thesis. http://arxiv.org/pdf/1004.1845

[8] Brünnler, K., Studer, T.: Syntactic cut-elimination for common knowledge. Annals of Pure and Applied Logic 160(1), 82-95 (2009)

[9] Bruscoli, P., Guglielmi, A.: On the proof complexity of deep inference. ACM Transactions on Computational Logic 10(2) (2009)

[10] Bruscoli, P., Guglielmi, A., Gundersen, T., Parigot, M.: A quasipolynomial cutelimination procedure in deep inference via atomic flows and threshold formulae. In: Clarke, E.M., Voronkov, A. (eds.) Logic for Programming, Artificial Intelligence, and Reasoning - 16th International Conference, LPAR-16. Lecture Notes in Computer Science (LNCS), vol. 6355, pp. 136-153. Springer (2010)

[11] Crocco, G., Lamarre, P.: On the connection between non-monotonic inference systems and conditional logics. In: Nebel, B., Sandewall, E. (eds.) Principles of Knowledge Representation and Reasoning: Proceedings of the 3rd International Conference KR'92. pp. 565-571 (1992)

[12] Fitting, M.: Prefixed tableaus and nested sequents. Annals of Pure Applied Logic 163(3), 291-313 (2012)

[13] Genovese, V., Giordano, L., Gliozzi, V., Pozzato, G.L.: A conditional constructive logic for access control and its sequent calculus. In: Brünnler, K., Metcalfe, G. 
(eds.) TABLEAUX 2011 (20th International Conference on Automated Reasoning with Analytic Tableaux and Related Methods). Lecture Notes in Artificial Intelligence (LNAI), vol. 6793, pp. 164-179. Springer-Verlag, Bern, Switzerland (July 2011)

[14] Genovese, V., Giordano, L., Gliozzi, V., Pozzato, G.L.: Logics in access control: A conditional approach. Journal of Logic and Computation p. to appear (2012)

[15] Gent, I.P.: A sequent or tableaux-style system for lewis's counterfactual logic vc. Notre Dame Journal of Formal Logic 33(3), 369-382 (1992)

[16] Ginsberg, M.L.: Counterfactuals. Artificial Intelligence 30(1), 35-79 (1986)

[17] Giordano, L., Gliozzi, V., Olivetti, N., Pozzato, G.L.: Analytic Tableaux Calculi for KLM Logics of Nonmonotonic Reasoning. ACM Trans. Comput. Logic 10(3) (2009)

[18] Giordano, L., Gliozzi, V., Olivetti, N.: Weak AGM postulates and strong ramsey test: A logical formalization. Artificial Intelligence 168(1-2), 1-37 (2005)

[19] Giordano, L., Gliozzi, V., Olivetti, N., Schwind, C.: Tableau calculus for preference-based conditional logics: Pcl and its extensions. ACM Trans. Comput. Logic 10(3) (2009)

[20] Goré, R., Postniece, L., Tiu, A.: Cut-elimination and proof-search for biintuitionistic logic using nested sequents. In: Areces, C., Goldblatt, R. (eds.) Advances in Modal Logic. vol. 7, pp. 43-66. College Publications (2008), http: //www.aiml. net/volumes/volume7/Gore-Postniece-Tiu.pdf

[21] Grahne, G.: Updates and counterfactuals. Journal of Logic and Computation 8(1), 87-117 (1998)

[22] Kraus, S., Lehmann, D., Magidor, M.: Nonmonotonic reasoning, preferential models and cumulative logics. Artificial Intelligence 44(1-2), 167-207 (1990)

[23] Lehmann, D., Magidor, M.: What does a conditional knowledge base entail? Artificial Intelligence 55(1), 1-60 (1992)

[24] Lellmann, B., Pattinson, D.: Sequent Systems for Lewis' Conditional Logics. In: Luis Fariñas del Cerro, Andreas Herzig, J.M. (ed.) Logics in Artificial Intelligence - 13th European Conference, JELIA 2012. Lecture Notes in Artificial Intelligence (LNAI), vol. 7519, p. to appear. Springer-Verlag, Toulouse, France (Sptember 2012)

[25] Lewis, D.: Counterfactuals. Basil Blackwell Ltd (1973)

[26] Nute, D.: Topics in conditional logic. Reidel, Dordrecht (1980)

[27] Nute, D.: Conditional logic. In: Handbook of Philosophical Logic, Vol. II, pp. 387-439. F. Guenthner and D. Gabbay, D. Reidel Publishing Company, Dordrecht, Holland (1984) 
[28] Olivetti, N., Pozzato, G.L., Schwind, C.B.: A Sequent Calculus and a Theorem Prover for Standard Conditional Logics. ACM Transactions on Computational Logic (ToCL) 8(4) (2007)

[29] Pattinson, D., Schröder, L.: Generic modal cut elimination applied to conditional logics. Logical Methods in Computer Science 7(1) (2011)

[30] Schröder, L., Pattinson, D., Hausmann, D.: Optimal tableaux for conditional logics with cautious monotonicity. In: ECAI. pp. 707-712 (2010)

[31] Stalnaker, R.: A theory of conditionals. In N. Rescher (ed.), Studies in Logical Theory, American Philosophical Quarterly, Monograph Series no.2, Blackwell, Oxford pp. 98-112 (1968)

[32] de Swart, H.C.M.: A gentzen- or beth-type system, a practical decision procedure and a constructive completeness proof for the counterfactual logics vc and vcs. Journal of Symbolic Logic 48(1), 1-20 (1983) 